\title{
Efficient probabilistic inversion using the rejection sampler-exemplified on airborne EM data
}

\author{
Thomas M. Hansen ${ }^{\circledR}$ \\ Department of Geoscience, Aarhus University, Høegh-Guldbergs Gade 2, 8000 Aarhus C, Denmark. E-mail: tmeha@geo.au.dk
}

Accepted 2020 October 9. Received 2020 October 7; in original form 2020 April 16

\begin{abstract}
SUMMAR Y
Probabilistic inversion methods, typically based on Markov chain Monte Carlo, exist that allow exploring the full uncertainty of geophysical inverse problems. The use of such methods is though limited by significant computational demands, and non-trivial analysis of the obtained set of dependent models. Here, a novel approach, for sampling the posterior distribution is suggested based on using pre-calculated lookup tables with the extended rejection sampler. The method is (1) fast, (2) generates independent realizations of the posterior, and (3) does not get stuck in local minima. It can be applied to any inverse problem (and sample an approximate posterior distribution) but is most promising applied to problems with informed prior information and/or localized inverse problems. The method is tested on the inversion of airborne electromagnetic data and shows an increase in the computational efficiency of many orders of magnitude as compared to using the extended Metropolis algorithm.
\end{abstract}

Key words: Non-linear electromagnetics; Inverse theory; Numerical modelling; Statistical methods.

\section{INTRODUCTION}

An inverse problem refers to the problem of inferring information about a model (typically the subsurface) from direct (such as based on expected structures and parameter values) and indirect (such as based on geophysics) information. One can crudely divide methods for solving inverse problems into two groups: deterministic and probabilistic. The goal of deterministic methods is to infer one optimal model, see, for example, Hansen \& O'Leary (1993) and Menke (2012). Deterministic methods are typically computationally efficient and can be applied to large data sets, but most often lack the ability to explore the full space of uncertainty and cannot account for complex prior information.

The goal of probabilistic methods is to combine all available information in a probabilistic model (a probability distribution), consistent with all available information. The solution to a probabilistic formulation of an inverse problem is a probability distribution, the posterior probability distribution $\sigma(\mathbf{m})$ (Tarantola \& Valette 1982b). Except for linear Gaussian inverse problems (Tarantola \& Valette 1982a) an analytical description of $\sigma(\mathbf{m})$ is not feasible. Instead Markov chain Monte Carlo (McMC) based sampling methods exist that allow sampling of $\sigma(\mathbf{m})$ (Metropolis et al. 1953; Hastings 1970; Mosegaard \& Tarantola 1995). While guaranteed to in principle sample the correct posterior distribution, such sampling methods become both computationally expensive, to the point where they cannot be practically applied, and the results difficult to interpret, see, for example, Cordua et al. (2012), Zunino et al. (2014), Vats et al. (2015) and De Pasquale et al. (2019).
Several methods have been proposed to alleviate these challenges. For example, gradient-based methods such as Hamiltonian McMC and stochastic Newton methods (Neal et al. 2011; Martin et al. 2012) and parallel tempering has been suggested (Earl \& Deem 2005; Sambridge 2013). While these methods may be more efficient than more simple McMC sampling methods for some problems, they are still so computationally demanding, that it limits its use in practice.

Here, we present a method, relying the on the extended rejection sampling algorithm, that allows (1) very fast sampling of independent realizations of $\sigma(\mathbf{m}),(2)$ analysis the posterior probability distribution and (3) the use of arbitrarily complex prior information. It is based on using lookup tables of pre-computed sets of models and data. As the lookup table is limited in size, this may lead to a limit of the best resolution that can be achieved. This can be quantified and accounted for as a modelling error on the data.

First, the theory behind the method is outlined. Then, it is applied to a probabilistic inversion of airborne EM data, and compared to inversion results obtained using McMC sampling.

\section{THEORY}

Let $\mathbf{m}$ represent some model parameters describing physical parameters. In the following example, $\mathbf{m}$ represents subsurface logresistivity. Let $\mathbf{d}$ refer to the, noise-free, physical response of $\mathbf{m}$, through $g(\mathbf{m}) . n(\mathbf{m})$ refer to the noise (due to both measurement 
and modelling errors) related to a specific observed data $\mathbf{d}_{\mathrm{obs}}$, that can be described through the following process:

$\mathbf{d}_{\mathrm{obs}}=g(\mathbf{m})+n(\mathbf{m})=\mathbf{d}+n(\mathbf{m})$,

The forward problem (i.e. solving $\mathbf{d}=g(\mathbf{m})$ ) is widely studied, and many different solutions exist for many types of forward problems. For example, the electromagnetic (EM) response to a resistivity field can be computed in 1-D, 2-D and 3-D. The opposite problem, the inverse problem, is more challenging but allows the inference of the model parameters (that we cannot directly assess) from indirect measurements.

Using a deterministic approach to inverse problems, the goal is to find one optimal model, given some specific choice of regularization, see, for example, Menke (2012). This can be for example the simplest model that fits the data within the noise (Constable et al. 1987). Here, we consider the probabilistic approach to inverse problems.

\subsection{Probabilistic inverse problems}

Tarantola \& Valette (1982b) developed a probabilistic approach to solving inverse problems, in which all information has to be quantified probabilistically through a probability distribution or a likelihood. Typically, available information consists of so-called prior information and information from geophysical data.

$\rho(\mathbf{m})$ is the probability distribution representing prior knowledge about the model parameters. This can come from nearby outcrops, geological expert knowledge, previous surveys and similar sources.

Information from geophysical data is quantified by the likelihood function, $L$, which is a probability distribution that quantifies the expected data residual $L\left(\mathbf{d}_{\mathrm{obs}}-\mathbf{d}\right)$. For notational brevity, we will refer to the likelihood function as $L(\mathbf{m})=L\left(\mathbf{d}_{\text {obs }}-g(\mathbf{m})\right)$.

Once the available information is quantified, the combined state of information (in form of the prior distribution and the likelihood) can be obtained using the concept of conjunction of states of information (Tarantola \& Valette 1982b), which results in the posterior probability distribution:

$\sigma(\mathbf{m})=k \rho(\mathbf{m}) L(\mathbf{m})$

where

$k^{-1}=\int \rho(\mathbf{m}) L(\mathbf{m}) d \mathbf{m}$

is a normalizing constant ensuring that $\int \sigma(\mathbf{m}) d \mathbf{m}=1$.

A general probabilistic description of the likelihood function is given as (Tarantola (2005))

$L(\mathbf{m})=\int_{\mathcal{D}} \frac{\rho_{D}(g(\mathbf{m})) \theta(\mathbf{d} \mid \mathbf{m})}{\mu_{D}(\mathbf{d})}$,

where $\rho_{D}(\mathbf{d})$ describes measurement uncertainties, typically related to the instrument recording the data. $\theta(\mathbf{d} \mid \mathbf{m})$ describes the modelling error and is a probabilistic description of the uncertainty related to evaluation of the forward problem on a model $\mathbf{m} . \mu_{D}(\mathbf{d})$ is the homogeneous probability distribution (see Tarantola 2005 for more details).

If the measurement uncertainty is Gaussian, $\rho_{D}(\mathbf{d}) \sim \mathcal{N}\left(\mathbf{0}, \mathbf{C}_{\mathrm{d}}\right)$, and the modelling errors also Gaussian, $\theta(\mathbf{d} \mid \mathbf{m})=\mathcal{N}\left(\mathbf{d}_{\mathrm{t}}, \mathbf{C}_{\mathrm{t}}\right)$, then the likelihood becomes Gaussian (Mosegaard \& Tarantola 2002):

$$
\begin{aligned}
L(\mathbf{m})= & \left((2 \pi)^{2}\left|\mathbf{C}_{\mathrm{d}}+\mathbf{C}_{\mathrm{t}}\right|\right)^{-.5} \exp \left(-\frac{1}{2}\left(\left(\mathbf{d}-g(\mathbf{m})-\mathbf{d}_{\mathrm{t}}\right)^{\top}\right)\right. \\
& \left.\times\left(\mathbf{C}_{\mathrm{d}}+\mathbf{C}_{\mathrm{t}}\right)^{-1}\left(\mathbf{d}-g(\mathbf{m})-\mathbf{d}_{\mathrm{t}}\right)\right)
\end{aligned}
$$

In many cases, the modelling error is ignored (i.e. described by a delta function) in which case the likelihood is equivalent to the measurement uncertainty as $L(\mathbf{m})=\rho_{D}(g(\mathbf{m}))$. However, the uncertainty related to the forward modelling errors may be significant and higher than the measurement uncertainty (Hansen et al. 2014). Modelling errors will play an important role in the following, therefore we consider the full description of the likelihood in eq. (4), using the Gaussian assumption leading to eq. (5).

\subsection{Sampling the posterior probability distribution}

Except for linear Gaussian inverse problems (Tarantola \& Valette 1982a), it is typically not possible to describe $\sigma(\mathbf{m})$ analytically. Instead, sampling methods can be used to generate a sample of $\sigma(\mathbf{m})$, which is a collection of models (realizations) distributed according to $\sigma(\mathbf{m})$. Statistical properties of $\sigma(\mathbf{m})$ can then be analysed from the sample of $\sigma(\mathbf{m})$.

\subsubsection{Variants of the Metropolis-Hastings algorithm}

The most widely used algorithms to sample from $\sigma(\mathbf{m})$ are McMC methods based on variants of the Metropolis and MetropolisHastings algorithms (Metropolis et al. 1953; Hastings 1970). The algorithms are simple to implement and consist of iteratively running (1) an exploratory step (in which a model is proposed, $\mathbf{m}_{\text {pro, }}$, by perturbing a current model in the Markov chain, $\mathbf{m}_{\text {cur }}$ ), and (2) an exploitation step, in which it is decided through an acceptance probability, $P_{\text {acc }}$, whether to accept the proposed model as a new current model in the Markov chain. For the Metropolis algorithm (using a symmetrical proposal distribution in the exploitation step), the acceptance probability becomes (Metropolis et al. 1953)

$P_{\mathrm{acc}}=\frac{\sigma\left(\mathbf{m}_{\mathrm{pro}}\right)}{\sigma\left(\mathbf{m}_{\mathrm{cur}}\right)}$.

The only requirement for running the Metropolis algorithm is thus that one must be able to evaluate the posterior probability distribution on any given model $\mathbf{m}$. It is guaranteed that if run for a finite (perhaps long) time, the collection of models obtained from the Metropolis algorithm will reflect a sample of $\sigma(\mathbf{m})$.

In practice, though the use of the Metropolis algorithm is associated with some significant challenges. The computational requirements for running the Metropolis algorithm can be very high, even for quite low-dimensional and simple inverse problems. Further, it is not trivial to determine when a Markov chain has burned-in, such that the posterior distribution is sampled. The sequence of model realizations generated by the Metropolis algorithm does not represent independent realizations. It is not trivial to determine the number of iterations needed to obtain independent realizations and hence not trivial to determine when enough iterations have been performed (Kass et al. 1998). It is also not trivial to determine whether the full space of models has been considered, or whether the sampling algorithm is stuck in a local area of high posterior probability, see, for example, Ripley (2009). These challenges have been widely studied, and several methods have been proposed to alleviate the computational burden of McMC sampling methods, such as gradient-based proposal models and parallel tempering.

\subsubsection{Parallel tempering}

To avoid being trapped in a local minimum, the use of parallel tempering has been suggested (Earl \& Deem 2005; Sambridge 2013). 
Here, several McMC chains are run in parallel at different temperatures, which essentially scale the uncertainty and allow each chain to be more or less exploratory. This allows exploring more of the model parameter space and allows for, in principle, transitions between different areas of local minima located relatively far from each other. This, of course, is related to increased computational demands, and choosing the optimal number, and temperatures, of chains, may be non-trivial.

\subsubsection{Gradient-based McMC}

Hamiltonian McMC methods make use of the gradient of $\sigma(\mathbf{m})$ to propose new models, to obtain more efficient sampling (Neal et al. 2011). Stochastic Newton methods are related but use the second-order derivative of $\sigma(\mathbf{m})$, and are usually computationally more demanding (Martin et al. 2012). Most often these methods are applied in cases where a simple uniform prior model is used, such that the derivative of the posterior distribution is equal to the derivative of the likelihood function, which can be efficiently computed using, for example, the adjoint method for some geophysical problems (Tarantola 1986; Tromp et al. 2005; Plessix 2006; Fichtner et al. 2018). If the prior model is more complex, the gradient-based method for proposing new models cannot be readily used, as it may become intractable, to compute the derivatives of the posterior distribution. Even when these gradient-based sampling methods can be used, they require some user interaction by setting some tuning parameters, though some more automated approaches have been suggested (Hoffman \& Gelman 2014).

\subsubsection{Approximate forward modelling}

Evaluation of the likelihood, that requires an evaluation of the forward model, is often the computationally most expensive part of running the Metropolis algorithm. Therefore, one can make use of approximate forward models that are computationally much faster, such as based on fast to evaluate neural networks (Hansen \& Cordua 2017; Conway et al. 2019; Moghadas et al. 2020). This will lead to a modelling error that in some cases can be quantified and accounted for (Hansen et al. 2014; Köpke et al. 2018).

\subsubsection{The extended Metropolis algorithm}

A variant of the Metropolis algorithm is the extended Metropolis algorithm proposed by Mosegaard \& Tarantola (1995). It relies on the existence of an algorithm that samples the prior distribution $\rho(\mathbf{m})$ using a random walk, and a way to evaluate the likelihood, eq. (4). In the two-step sampling algorithm, the exploratory step consists of perturbing a current model, $\mathbf{m}_{\text {cur }}$, to a proposed model, $\mathbf{m}_{\text {pro }}$. The perturbation must be such that if iterating only the exploratory step, this should result in sampling the prior. The exploitation step consists of accepting the move to the proposed model with acceptance probability

$P_{\mathrm{acc}}=\frac{L\left(\mathbf{m}_{\mathrm{pro}}\right)}{L\left(\mathbf{m}_{\mathrm{cur}}\right)}$.

A key property of the extended Metropolis algorithm is that it allows the use of arbitrarily complex prior information, as long as an algorithm exists that can sample it. Also, the acceptance probability requires only that the likelihood can be evaluated eq. (7), and not the full posterior as when using the classical Metropolis algorithm, eq. (6). If informed prior information exists, and can be sampled, then the extended Metropolis algorithm may provide an efficient approach to sampling the posterior as opposed to using the classical Metropolis algorithm, while it is still computationally demanding (Cordua et al. 2012; Zunino et al. 2014). A detailed discussion about the extended Metropolis algorithm can be found in Mosegaard \& Tarantola (1995) and Hansen et al. (2016).

\subsubsection{The rejection sampling algorithm}

The rejection sampler is a very simple, perhaps the simplest, alternative to the Metropolis algorithm for sampling a probability distribution, here $\sigma(\mathbf{m})$.

Let $h(\mathbf{m})$ refer to a proposal distribution from which a realization can be generated (preferably in a computationally efficient manner), and for which $h(\mathbf{m})>0$ for all $\mathbf{m}$ where $\sigma(\mathbf{m})>0$. In other words, any model with non-zero posterior probability distribution value, must have a non-zero probability of being realized from the proposal distribution. Then, the rejection sampler can be implemented as follows in order to sample the posterior distribution:

1. Propose a model $\mathbf{m}_{\text {pro }}$, as a realization of $h(\mathbf{m})$.

2. Accept this model as a realizations of $\sigma(\mathbf{m})$ with probability $P_{\text {acc }}$

$P_{\text {acc }}=\frac{\sigma\left(\mathbf{m}_{\text {pro }}\right)}{h\left(\mathbf{m}_{\text {pro }}\right) k}$.

$k$ is a normalizing constant that ensures $P_{\text {acc }} \leq 1$ for all $\mathbf{m}$. Here, $k \geq \max (\sigma(\mathbf{m}) / h(\mathbf{m}))$, that is, $k$ must be larger than the maximum possible value of $\sigma(\mathbf{m}) / h(\mathbf{m})$. The better the proposal distribution $h(\mathbf{m})$ resembles $\sigma(\mathbf{m})$, the more effecient the rejection sampler will be. Often the proposal distribution is assumed to be the uniform distribution, in which the acceptance probability becomes

$P_{\mathrm{acc}}=\frac{\sigma\left(\mathbf{m}_{\mathrm{pro}}\right)}{k}$.

where, $k \geq \max (\sigma(\mathbf{m}))$.

Implementation of the rejection sampler algorithm using eq. (9) is extremely simple, and requires only that (1) one can sample from a uniform distribution, (2) one can evaluate the posterior probability distribution in any model and (3) that a normalization constant $k$ can be estimated. Any model that gets accepted using this algorithm, will by construction represent an independent realization of $\sigma(\mathbf{m})$.

However, the rejection sampler is rarely used in practice as it is considered applicable for only low-dimensional inverse problems with few model parameters due to the 'great emptiness of largedimensional spaces' (Tarantola 2005) which suggest that most proposed models will be rejected. In addition it is in practice non-trivial to choose the normalization constant $k$ in eqs (8) and (9). Choosing a high value leads to a less efficient algorithm while choosing a too low value leads to an algorithm that does not sample the correct posterior distribution. For directly sampling, the posterior distribution it is for most practical cases wildly inefficient.

\subsubsection{The extended rejection sampling algorithm}

The extended rejection sampler is a simple variant of the rejection sampler, as it samples the product of two probability distributions, here $\sigma(\mathbf{m}) \propto \rho(\mathbf{m}) L(\mathbf{m})$, as the extended Metropolis algorithm (Hansen et al. 2016). It requires that one can sample from one probability distribution, here $\rho(\mathbf{m})$, and that one can evaluate other probability distribution, here $L(\mathbf{m})$. To sample from the posterior distribution, one can use the traditional rejection sampler with the 
prior distribution as proposal distribution, $h(\mathbf{m})=\rho(\mathbf{m})$, leading to the extended rejection sampler:

1. Propose a model $\mathbf{m}_{\text {pro }}$, as a realization from $\rho(\mathbf{m})$.

2. Accept this model with probability $P_{\text {acc }}$

$P_{\mathrm{acc}}=\frac{\sigma\left(\mathbf{m}_{\mathrm{pro}}\right)}{h\left(\mathbf{m}_{\mathrm{pro}}\right) k}=\frac{\rho\left(\mathbf{m}_{\mathrm{pro}}\right) L\left(\mathbf{m}_{\mathrm{pro}}\right)}{\rho\left(\mathbf{m}_{\mathrm{pro}}\right) k}=\frac{L\left(\mathbf{m}_{\mathrm{pro}}\right)}{k_{L}}$,

where the $k_{L}$ is a normalizations constant that must be higher than the maximum possible value of $L(\mathbf{m}), K_{L} \geq \max (L(\mathbf{m}))$. The algorithm will be most efficient when $k_{L}=\max (L(\mathbf{m}))$. Applying this algorithm ensures that accepted models represent independent realizations of $\sigma(\mathbf{m})$. Implementation requires that (1) one can sample from $\rho(\mathbf{m}),(2)$ one can evaluate the likelihood and (3) that one can estimate the normalization constant $k_{L}$. This means that, as for the extended Metropolis algorithm, the extended rejection sampler can be used to sample a posterior probability distribution for which the prior probability distribution cannot be evaluated (and hence nor the posterior distribution), but where an algorithm exists that allows sampling from the prior distribution. This allows exploring a broader range of inverse problems using the extended rejection sampler as opposed to the traditional rejection sampler.

If realizations of the prior can be efficiently generated, then the extended rejection sampler may be much more efficient than the classical rejection sampling algorithm, as the use of the prior potentially limits the emptiness of the space to be considered by the rejection sampler.

The dimension of the reduced space to be explored by sampling directly from the prior can be quantified through the 'number of effective free parameters' as discussed by Hansen (2020), that quantifies the average minimum number of model parameters $N_{\text {free }}$ that needs to be inferred to completely represent a realization of a specific probability distribution. Say $\mathbf{m}$ consist of $N_{m}$ model parameters. Then, using the traditional rejection sampler with a uniform proposal distribution leads to a space to be explored by the rejection sampler of dimension $N_{\text {free }}=N_{m}$. But in case using the extended rejection sampler with an informed prior model, then the effective dimension can be reduced to $N_{\text {free }} \leq N_{m}$. Geostatistical models can be used to quantify prior information for inverse problems, see, for example, Bosch et al. (2010) and Hansen et al. (2012). Geostatistical models describe spatial correlations between model parameters and hence represent cases of informed prior models, where $N_{\text {free }}$ can be much smaller than $N_{m}$. When using the extended rejection sampler with such informed prior models, that is, where $N_{\text {free }}<<N_{m}$, the effective space to be considered by the extended rejection sampler can be much lower dimensional than when using the rejection sampler with a uniform proposal distribution. For certain spatial models, $N_{\text {free }}$ can be computed, and hence the complexity of the sampling problem can be quantified (Hansen 2020).

Still, the extended rejection sampler is typically much more CPU demanding than the extended Metropolis algorithm.

The method outlined below is designed to sample, and compute summary statistics of, the posterior distribution in a fraction of the time needed to use the traditional sampling approaches listed above, and will be compared to the results presented in Hansen \& Minsley (2019).

\section{APPROXIMATE EXTENDED REJECTION SAMPLING USING LOOKUP TABLES}

Here, an alternative application of the extended rejection sampler is proposed that does not use the sequential approach described above, but instead uses a pre-computed set of prior model realizations and corresponding data responses. The method can be implemented as:

A. Build lookup tables

B. Compute modelling error (optional)

C. Apply the extended rejection sampler with lookup tables

The core of the algorithm consists of applying steps A and C. Step B is optional and will be discussed later.

\subsection{A. Create a lookup table with pre-computed models and data}

First, $N_{\text {lu }}$ realizations of the prior are generated as

$\mathbf{M}^{*}=\left[\mathbf{m}_{1}^{*}, \mathbf{m}_{2}^{*}, \ldots, \mathbf{m}_{N_{l u}}^{*}\right]$.

The corresponding data for each realization of the prior is computed using the forward model $\mathbf{d}_{i}^{*}=g\left(\mathbf{m}_{i}^{*}\right)$, to obtain

$\mathbf{D}^{*}=\left[\mathbf{d}_{1}^{*}, \mathbf{d}_{2}^{*}, \ldots, \mathbf{d}_{N_{l u}}^{*}\right]$.

This constitutes a training data set $\left[\mathbf{M}^{*} ; \mathbf{D}^{*}\right]$ stored in a lookup table, preferably in fast to access memory.

For a specific choice of parametrization, prior and forward model, this needs only be done once. Further, the prior can be arbitrarily complex. The only requirements are that one must be able to generate a large sample of the prior and solve the forward problem for each stored model. For example, any geostatistical simulation method can be used to quantify prior information (Deutsch \& Journel 1992; Mariethoz \& Caers 2014).

\subsection{Extended rejection sampling with lookup tables}

Given an observed set of data $\mathbf{d}_{\text {obs }}$, the extended rejection sampler can now be evaluated by computing the likelihood for all $N_{\text {lu }}$ sets of data in the training data set using eq. (4). This task is typically much simpler than either sampling the prior or evaluating the forward model, and hence computationally much faster. The normalizing constant in eq. (10), $k_{L}=L_{\max }(\mathbf{m})$, is trivial to find from the $N_{\text {lu }}$ likelihood values.

Once the likelihood has been computed several independent realizations of the posterior distribution can be evaluated simply by drawing models from the training data set, proportional to the acceptance probability (which is the normalized likelihood in eq. 10). This is computationally trivial and will generate a sample of the posterior distribution $\sigma(\mathbf{m})$. Statistical properties such as the mean, variance and entropy can in a similar manner be efficiently computed.

\subsubsection{The choice of $N_{l u}$}

In practice, the lookup table will be of finite size, just as the number of iterations for any variant of the Metropolis algorithm or rejection sampler described above will be finite (and hence the number of considered models). For Metropolis-based algorithms, the number of iterations must be large enough that the posterior is being sampled (i.e. burn-in has been reached), and large enough 
that enough independent realization of $\sigma(\mathbf{m})$ has been generated. Rejection sampler-based algorithms have no issues with burn-in (assuming the normalization constant has been properly chosen), but enough iterations need to be performed to obtain enough independent realizations. For both cases, the value of 'enough' independent realizations depends on the use of these realizations.

Using the extended rejection sampler with lookup tables the relative frequency with which a model $\mathbf{m}_{i}^{*}$ from the lookup table is sampled is proportional to the likelihood of that model $L\left(\mathbf{m}_{i}^{*}\right)$, irrespective of the choice of $N_{\text {lu }}$. The main consideration of the choice of $N_{\mathrm{lu}}$ is that it should lead to a significant amount of models with significant acceptance probability and that some of these models should lead to fitting the observed data within the noise. These considerations could be attributed to the classical rejection sampler as well.

Specific for the extended rejection sampler with lookup tables, the use of a finite-sized lookup table leads to an upper limit of the resolution of the posterior that one can expect. Only models in the lookup table can be generated as realizations of the posterior. Say $\mathbf{m}_{\text {ref }}$ represents the true model, then the best possible representation of this model will be the most similar model in the lookup table, $\mathbf{m}_{\text {opt }}$. The difference $\delta \mathbf{m}=\mathbf{m}_{\text {ref }}-\mathbf{m}_{\text {opt }}$ represents the minimum discrepancy one can expect for using a lookup table of a certain size.

Below we propose to quantify this error in expected resolution as a function of the lookup table, in form of a Gaussian modelling error, to ensure that a small choice of $N_{\text {lu }}$ does not lead to inconsistent posterior statistics with too little variability, but instead to consistent statistics with less resolution than when using a higher value of $N_{\mathrm{lu}}$.

\subsection{B. Estimate the modelling error due to using a finite-sized lookup table.}

As discussed above, a finite-sized lookup table will lead to a limit to the resolution that can be expected, as the actual reference model one seeks to infer information about is not part of the lookup table.

This suggests that one should not fit the observed data better than what corresponds to this limit in resolution. Here a method is proposed that quantifies a Gaussian noise model that expresses the data error related to the expected limited accuracy of the models in the lookup table relative to the true model. We refer to this error as a modelling error, as it can be considered an error in the formulation of the model space that translates into modelling uncertainty on the data (Hansen et al. 2014). In short, we propose to simulate expected variability on the model parameters, by comparing a 'true model' to the corresponding 'most similar model' in the lookup table. This variability is then propagated to the data space using the forward model.

This potential modelling error is suggested quantified by constructing an approximate forward model $g_{\text {app }}$ that finds the forward response of a model $\mathbf{m}$ by finding the most similar model, $\mathbf{m}_{i_{o}}^{*}$, in the list of generated a priori models $\mathbf{M}^{*}$, such that

$\mathbf{d}_{\mathrm{app}}=g_{\mathrm{app}}(\mathbf{m})=g\left(\mathbf{m}_{i_{o}}^{*}\right)$

where

$\mathbf{m}_{i_{o}}^{*}=\min _{i=1,2, . ., N} \Pi\left(\mathbf{m}, \mathbf{m}_{i}^{*}\right)$,

where $\Pi\left(\mathbf{m}_{1}, \mathbf{m}_{2}\right)$ is a measure of the distance between two models $\mathbf{m}_{1}$ and $\mathbf{m}_{2}$. The specific distance measure used may dependent on the type of problem considered. The 'exact' forward model is still $\mathbf{d}=g(\mathbf{m})$.
The use of eq. (13) naturally introduces a modelling error as using $g_{\text {app }}$ is an approximation to using $g$. We suggest using the methodology in Hansen et al. (2014) to quantify the modelling error. It consists of first generating a sample of the modelling error, from which a Gaussian model of the modelling error is inferred.

A single realization of modelling error $\theta(\mathbf{d} \mid \mathbf{m})$ due to using $g_{\text {app }}$ as opposed to $g$, can be obtained simple by evaluating

$\delta \mathbf{d}_{i}=g\left(\mathbf{m}_{i}\right)-g_{\text {app }}(\mathbf{m})=\mathbf{d}_{i}-\mathbf{d}_{i \text { app }}$.

A full sample of $\theta(\mathbf{d} \mid \mathbf{m})$, consisting of $M$ realizations, can be obtained by repeating this process for $M$ independent realizations of the prior, $\mathbf{M}^{\rho}=\left[\mathbf{m}_{1}, \mathbf{m}_{2}, \ldots, \mathbf{m}_{M}\right]$. The forward response is evaluated for each of these models to also obtain the exact forward response, and then sample $\mathbf{D} \mathbf{D}^{*}$ of $\theta(\mathbf{d} \mid \mathbf{m})$ is given as

$$
\begin{aligned}
& \mathbf{D D}^{*}=\left[\mathbf{d}_{\mathbf{1}}-\mathbf{d}_{\mathbf{1} \text { app }},\right. \\
& \mathbf{d}_{\mathbf{2}}-\mathbf{d}_{\mathbf{2} \text { app }} \text {, } \\
& \left.\mathbf{d}_{\mathbf{M}}-\mathbf{d}_{\mathbf{M a p p}}\right]
\end{aligned}
$$

The mean $\mathbf{d}_{t}$ and covariance $\mathbf{C}_{t}$ can easily be computed from DD* (Hansen et al. 2014) such that

$\theta(\mathbf{d} \mid \mathbf{m}) \sim \mathcal{N}\left(\mathbf{d}_{\mathrm{t}}, \mathbf{C}_{\mathrm{t}}\right)$.

Then, the likelihood can be evaluated through eq. (5).

For very large training data sets (large $\left.N_{\mathrm{lu}}\right)$, the distance $\Pi\left(\mathbf{m}, \mathbf{m}_{i}^{*}\right)$ will be negligible, and hence also the modelling error will be negligible. Then, the extended rejection sampler with lookup tables will sample the full posterior distribution, and the modelling error need not be considered. For smaller training data sets (small $\left.N_{\text {lu }}\right)$, the distance $\Pi\left(\mathbf{m}, \mathbf{m}_{i}^{*}\right)$ will be significant and translate into a significant modelling error. In this case, an approximation to the full posterior distribution, with less resolution, will be sampled. The validity and accuracy of such an approximation of the posterior distribution are tested in the following case study. Note that $g_{\text {app }}$ is only used to quantify the modelling error. It is not otherwise a part of the inversion, where the full forward model $g(\mathbf{m})$ is used.

Below the extended rejection sampler with lookup tables will be applied and compared to using the extended Metropolis sampler for inversion of airborne EM data.

\section{PROBABILISTIC INVERSION OF AIRBORNE ELECTROMAGNETIC DATA}

Inversion of airborne EM data, with a 1-D prior and 1-D forward, is an example of a localized inverse problem. Often thousands of soundings (EM data measured at a specific location) are available as part of an airborne EM survey, and the same 1-D forward model and 1-D prior is considered for all data. This means that the extended rejection sampler with lookup tables can be utilized, while only computing the training data set once.

As an example, the same data and parametrization will be used as described in Hansen \& Minsley (2019), who used the extended Metropolis algorithm to sample the posterior distribution. They used 451 sounding from an airborne helicopter survey of frequencydomain EM data along an E-W trending profile from Morrill, Nebraska (Smith et al. 2010; Abraham et al. 2012). See fig. 12 in Minsley (2011) for an exact location. The noise of the EM data is assumed to be independent uncorrelated zero-mean Gaussian noise, 
with a standard deviation of 5 ppm (parts per million) plus 5 percent of the data value. For reference, Hansen \& Minsley (2019) find that generating a sample of a 1-D posterior distribution, consisting of 100 realizations, for one individual sounding, takes around 6 min (running 100000 iterations of the extended Metropolis sampler), and thus almost $2 \mathrm{~d}$ for 451 individual soundings. A typical airborne EM survey consists of many thousands of iterations, and therefore significant computational resources are needed to sample the posterior of such sets of thousands of 1-D inverse problems (Foks \& Minsley 2020).

\subsection{Single sounding 1-D case}

As an initial example, we consider a single sounding (number 121, at $x=6 \mathrm{~km}$ in fig. 12 in Minsley (2011). The prior is chosen to be the Gaussian type prior with a trimodal 1-D marginal distribution as argued by Hansen \& Minsley (2019) to be the best descriptive prior model based on nearby well logs (see a description of $\rho_{3}(\mathbf{m})$, in Hansen \& Minsley 2019 for more details).

The posterior distribution for the single sounding is inverted using both the extended Metropolis algorithm and the extended rejection sampler with lookup tables as demonstrated above.

For the extended rejection sampler with lookup tables training data sets of sizes $N_{\mathrm{lu}}=[1000,10000,100000,500000]$ are considered. The corresponding 1-D marginal distribution at each depth is shown in Fig. 1 for the four considered training data sample sizes. Figs 1(a)-(d) show the results in case quantifying and accounting for the modelling errors as described above. Figs 1(e)-(h) show the result obtained without modelling errors. For comparison, the extended Metropolis algorithm is run for 100000 iterations. The 1-D marginal of the corresponding posterior distribution is shown in Fig. 1(i).

Without accounting for modelling errors, the use of a small training data set $\left(N_{\mathrm{lu}}=1000,10000\right)$ results in biased posterior statistics with too little uncertainty (Figs $1 \mathrm{e}$ and $\mathrm{f}$ ). When accounting for modelling errors the results of using only $N_{\mathrm{lu}}=10000$ (Fig. 1b), is consistent with the results obtained using McMC sampling (Fig. 1i), except that more uncertainty is evident using the extended rejection sampler. Depending on the usecase, even using only $N_{\text {lu }}=1000$, seems to represent a useful estimate of $\sigma(\mathbf{m})$ (Fig. 1a). As $N_{\text {lu }}$ increases, the difference to the solution obtained using McMC sampling diminishes.

Fig. 2 shows both the time it takes to set up the lookup table (dashed line) and the time it takes to generate 100 independent realizations (solid line) for different choices of $N_{\text {lu }}$, with comparisons to running the extended Metropolis algorithm (dotted-dashed line).

Fig. 2 (dashed line) shows that it takes significant time to set up the lookup table. If one wishes to examine only one sounding for a specific choice of prior, and only do it once, then the extended Metropolis algorithm might be a better choice. Recall though that the computation of the lookup table needs only be done once, after which it can be reused.

When the lookup table is in place, Fig. 2 suggests that the extended rejection sampler using $N=500000$ is more than 700 times faster than using the extended Metropolis algorithm. Using $N=$ 10000 the speed up is around 250000 (more than 5 orders of magnitude).

Note that most of the computation time related to using the extended rejection sampler with lookup tables is related to computing the likelihood for each data set in the lookup tables. The computational demands to generate, in this case 100 realizations are negligible in comparison.

\subsection{1 soundings from Morrill, Nebraska}

Now all 451 soundings from the EW profile in Morrill, Nebraska, are considered. Using the extended Metropolis algorithm, 451 independent McMC algorithms are run, which suggest the computation time increase by a factor of 451 . In case using the extended rejection sampler with lookup tables, the pre-computed training data set can be reused, and only the likelihood needs to be computed for each sounding.

\subsubsection{Ignoring the modelling error}

Figs 3 and 4 show the pointwise mean and standard deviation along the profile, using the extended rejection sampler for $N_{\mathrm{lu}}=[1000$, $10000,100000,50000]$ in case ignoring the modelling error and compared to using the McMC algorithm, as in Hansen \& Minsley (2019).

A significant number of artefacts can be identified comparing the mean model obtained using the rejection sampler (Figs 3a-d), as opposed to using the extended Metropolis algorithm (Fig. 3e). The number of artefacts diminishes as the size of the lookup table increase. Using very large lookup tables, $N_{\text {lu }}=500000$ (Fig. 3d), the overall structure of the mean model obtained using the rejection sampler is similar to that of using the McMC.

The pointwise standard deviation (Fig. 4), quantify the certainty of the resistivity value inferred in a specific pixel, and highlight a significant problem: Not only are artefacts clearly visible in the mean models for $N_{\text {lu }}<500000$ (Figs 3a-c), they also appear to be well resolved as the standard deviation at the same location is very low (Figs $4 \mathrm{a}-\mathrm{c}$ ). The standard deviation increase as the $N_{\text {lu }}$ increases.

Thus, without accounting for the modelling error due to using a finite lookup table, the sample obtained using the extended rejection sampler with lookup tables should not be used in practice for small lookup tables, as it leads to biased estimates and too small posterior variability. The modelling error is most significant for smaller values of $N_{\mathrm{lu}}$, and therefore one may be able to use the rejection sampler for large values of $N_{\mathrm{lu}}$ without accounting for modelling errors.

\subsubsection{Accounting for the modelling error}

Figs 5 and 6 show the same pointwise mean and standard deviation along the profile, as in Figs 3 and 4, but in this case when quantifying and accounting for the modelling errors due to using a limited size lookup tables, as described above.

It is evident comparing Fig. 5 to Fig. 3 that fewer artefacts are visible in the mean models. The visible structures becomes sharper as $N_{\text {lu }}$ increases, but the results obtained even using $N_{\text {lu }}=1000$ could be useful, as a rough estimate of the subsurface mean resistivity. Using $N_{\text {lu }}=500000$, there is no significant difference between the results obtained using McMC (Fig. 5e), and the extended rejection sampler with lookup tables (Fig. 5d).

Contrary to the results shown in Fig. 4, the standard deviation decreases as $N_{\text {lu }}$ increases, when accounting for the modelling error.

Fig. 7 shows the average entropy for each sounding, computed from the pointwise standard deviation shown in Figs 4 and 6. 
(a) $\mathrm{N}_{\mathrm{lu}}=1000$

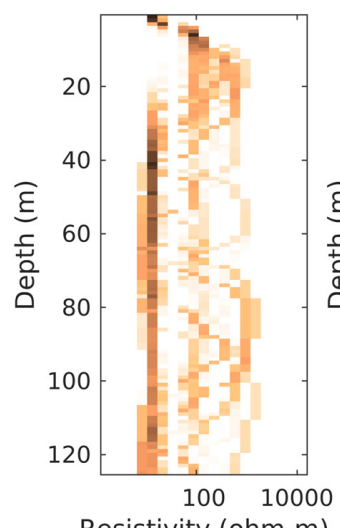

Resistivity (ohm-m)

(e) $\mathrm{N}_{\mathrm{lu}}=1000$

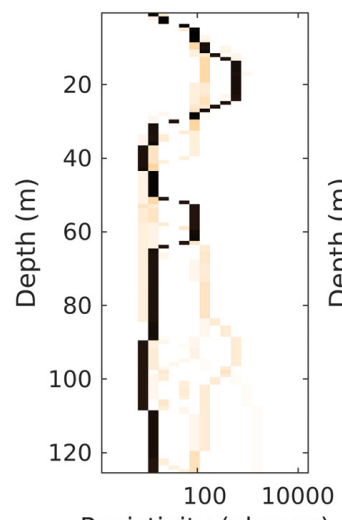

Resistivity (ohm-m) (b) $\mathrm{N}_{\mathrm{lu}}=10000$

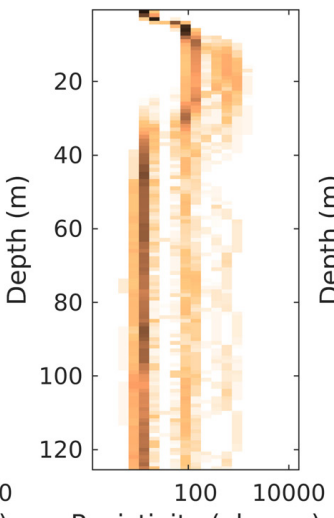

Resistivity (ohm-m)

(f) $\mathrm{N}_{\mathrm{lu}}=10000$

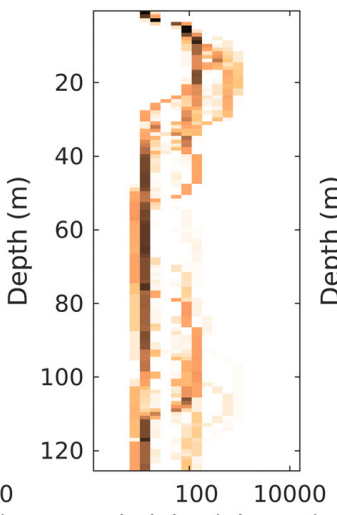

Resistivity (ohm-m) (c) $\mathrm{N}_{\mathrm{lu}}=100000$

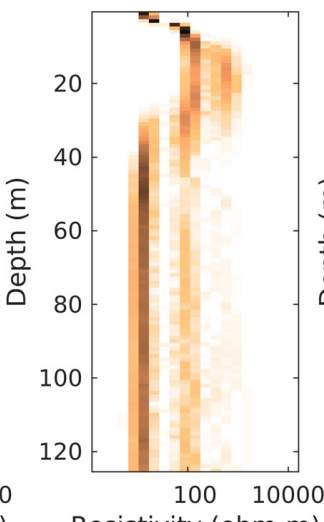

Resistivity (ohm-m)

(g) $N_{l u}=100000$

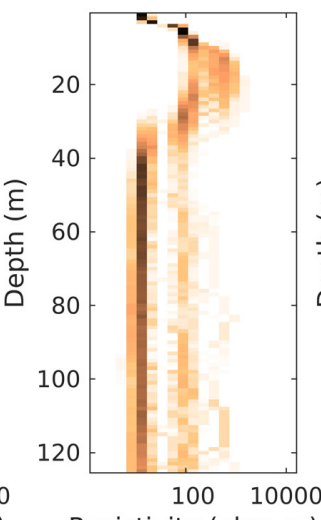

Resistivity (ohm-m) (d) $\mathrm{N}_{\mathrm{lu}}=500000$

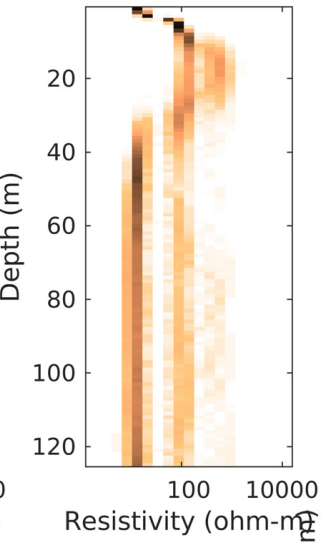

(h) $\mathrm{N}_{\mathrm{lu}}=500000$

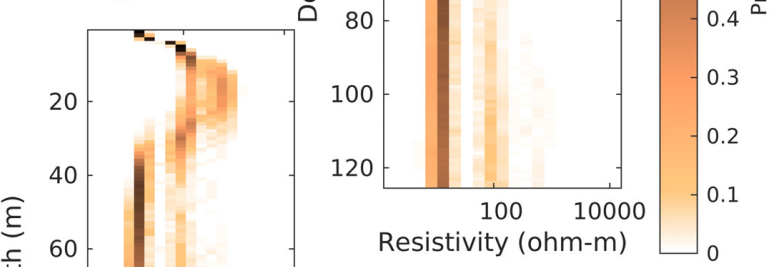

i) Metropolis

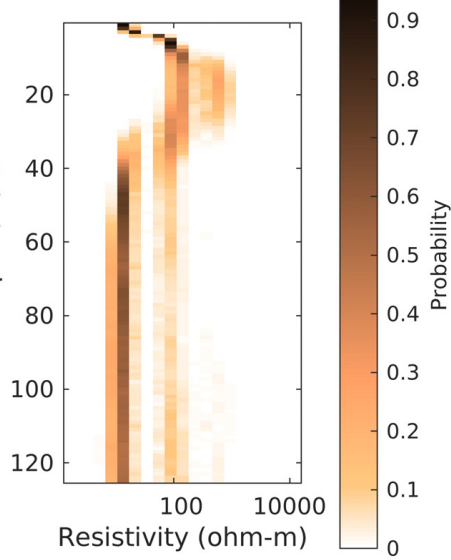

बin

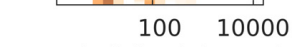

Resistivity (ohm-m)

Figure 1. 1-D marginal distributions obtained at $x=6 \mathrm{~km}$ using the extended rejection sampler with $N=[1000,10000,100000,500000]$, in cases (a)-(d) accounting for the modelling error, and (e)-(h) ignoring the modelling error. (i) Obtained using the extended Metropolis algorithm as in Hansen \& Minsley (2019).

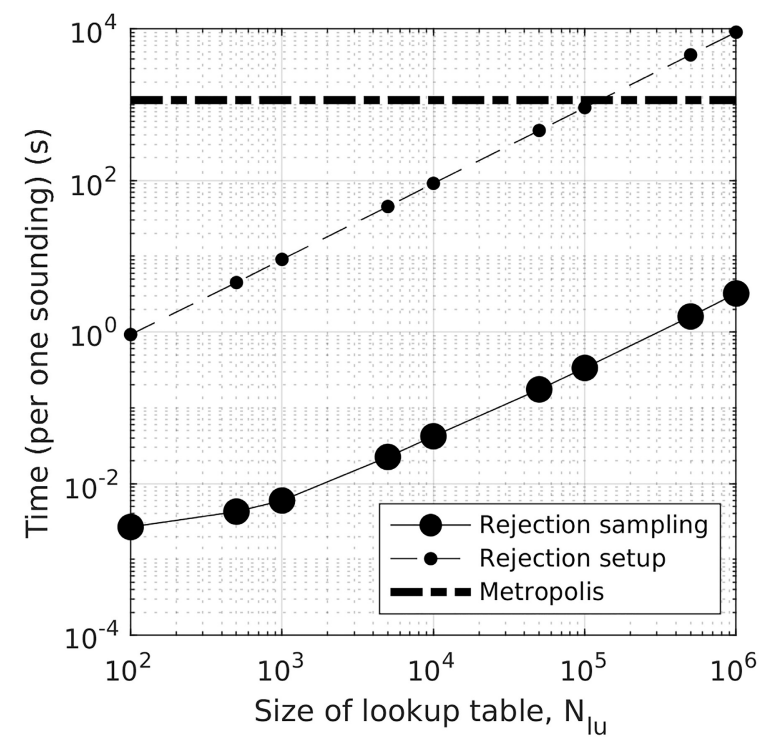

Figure 2. Time spend to set up the lookup table (dashed line), to sample the posterior for a single sounding using the extended rejection sampler (solid line, for different sizes of lookup table, $N_{\mathrm{lu}}$ ), and to sample the posterior using the extended Metropolis algorithm for one sounding (dashed-dotted line).
Fig. 7(a) shows that when accounting for the modelling errors, as $N_{\text {lu }}$ increases, the entropy decreases, that is, the information content increases. In all cases, the entropy of using the extended rejection sampler with lookup tables provides less information, more entropy, than using the extended Metropolis sampler, as expected when making use of the modelling error.

Fig. 7(b) shows that when ignoring the modelling errors, as $N_{\mathrm{lu}}$ increases, the entropy increases, i.e. the information content decreases! This is counterintuitive. For $N_{\text {lu }}=1000$ and 10000 , the entropy is most often much lower than that obtained using the extended Metropolis sampler. This is problematic and suggests that using smaller lookup tables and no modelling should be avoided. It does however also suggest that when using a large lookup table and no modelling error, the entropy is close to the entropy obtained using the extended Metropolis sampler.

Figs 8 and 9 show the Kullback-Leibler (KL) distance between the posterior probability distribution obtained using the extended Metropolis sampler and the different variants considered for the rejection sampler with lookup tables. Fig. 8 shows the KL distance for each model parameter, and Fig. 9 shows the average KL distance at each sounding location. The KL distance is a quantitative measure of similarity between two probability distributions.

It is clear from Fig. 8 that the similarity of the posterior obtained using the rejection sampler with lookup tables increase (the KL distance decrease) as the size of the lookup table increases. 
(a)

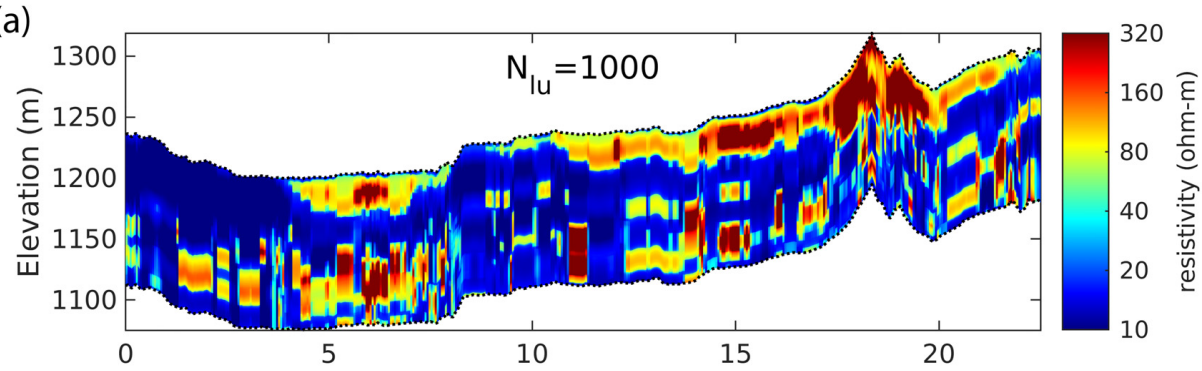

(b)

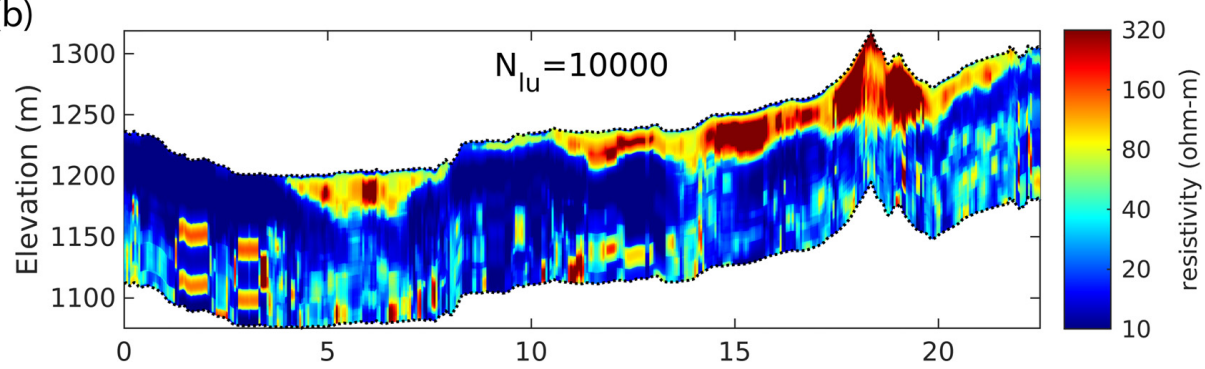

(c)

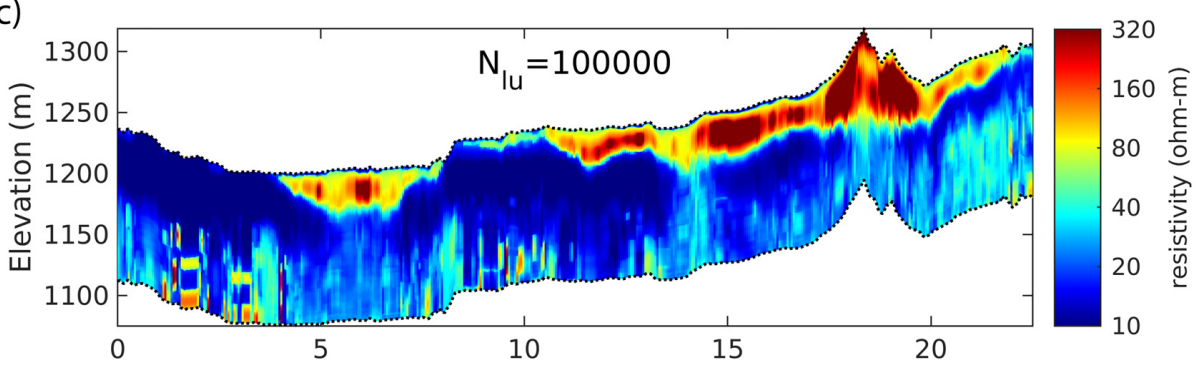

(d)

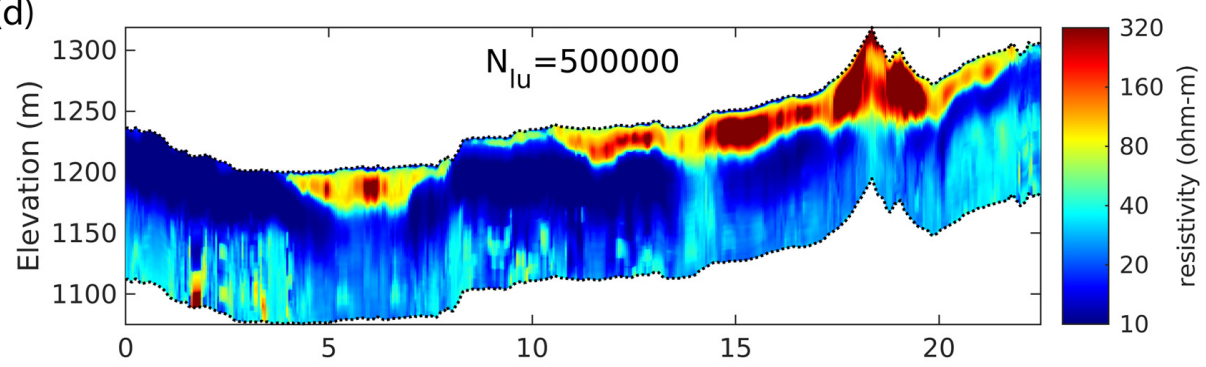

(d)

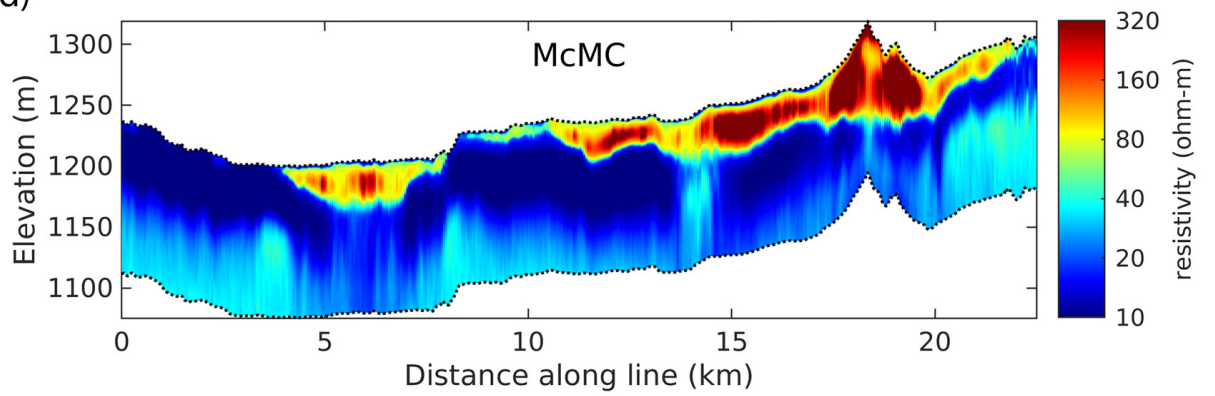

Figure 3. (a)-(d) Posterior mean obtained using $N_{\mathrm{lu}}=[1000,10000,100000,500000]$ without modelling error, and (e) McMC Metropolis sampling.

It is also clear that using smaller lookup tables, the posterior distribution obtained while accounting for the modelling error is much more similar to the posterior distribution obtained using the extended Metropolis sampler than when ignoring the modelling error.
Figs 7(b) and 9(b) demonstrate the one should not use the extended rejection sampler with a too-small lookup table and without accounting for modelling error, as it leads to an underestimation of the variability of the posterior distribution, and also cause a severe bias in the estimated mean model. If $N_{\text {lu }}$ is high enough (here 
(a)

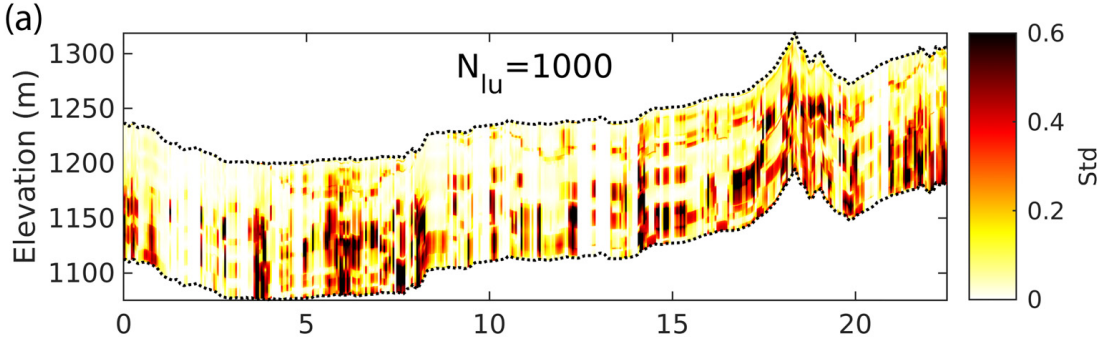

(b)
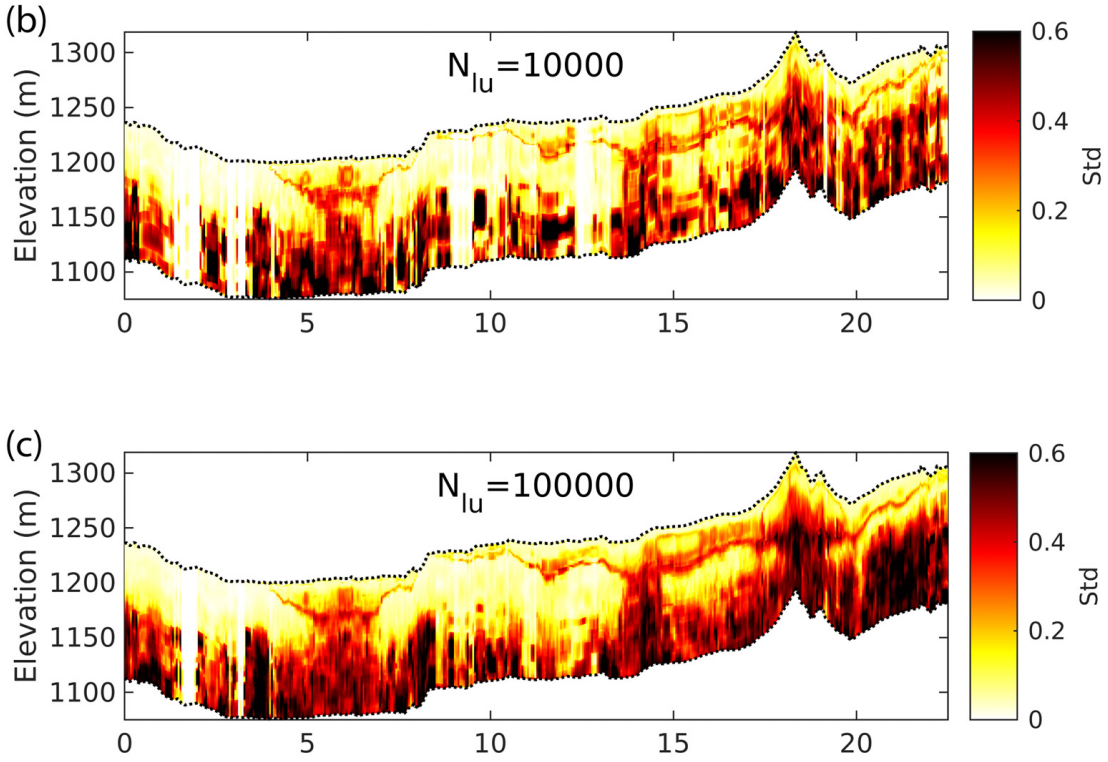

(d)

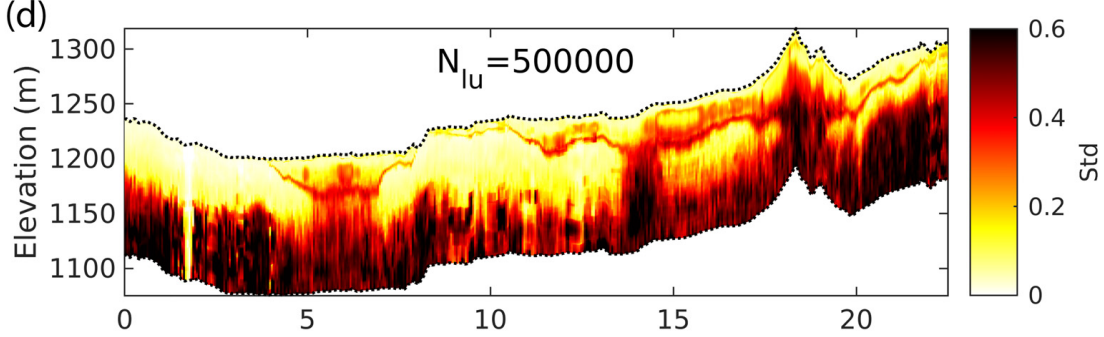

(d)

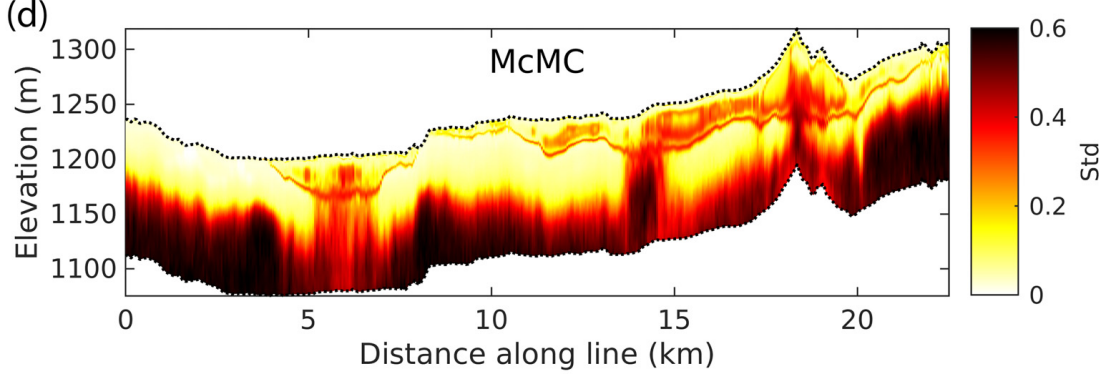

Figure 4. (a)-(d) Posterior standard deviation obtained using $N_{\mathrm{lu}}=[1000,10000,100000,500000]$ without modelling error, and (e) McMC Metropolis sampling.

when $N_{\text {lu }}>100000$ ) one can use the method. One way to find a useful number for $N_{\text {lu }}$ without using the modelling error is to keep increasing $N_{\text {lu }}$ until the entropy stops increasing and becomes stable.
When accounting for the modelling error, Figs 7(a) and 9(a) quantify that one can choose to make use of the extended rejection sampler, even with a smaller lookup table. This comes at the expected price of an increase in the entropy, reducing the information 
(a)

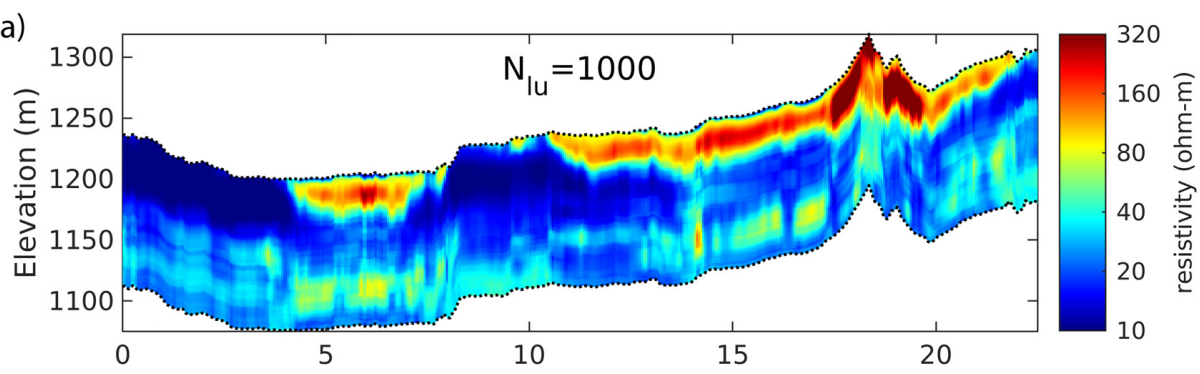

(b)

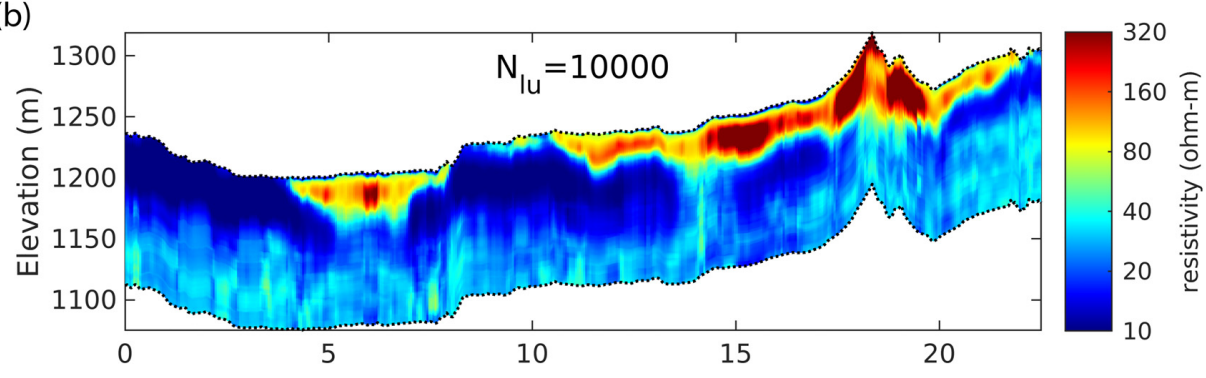

(c)
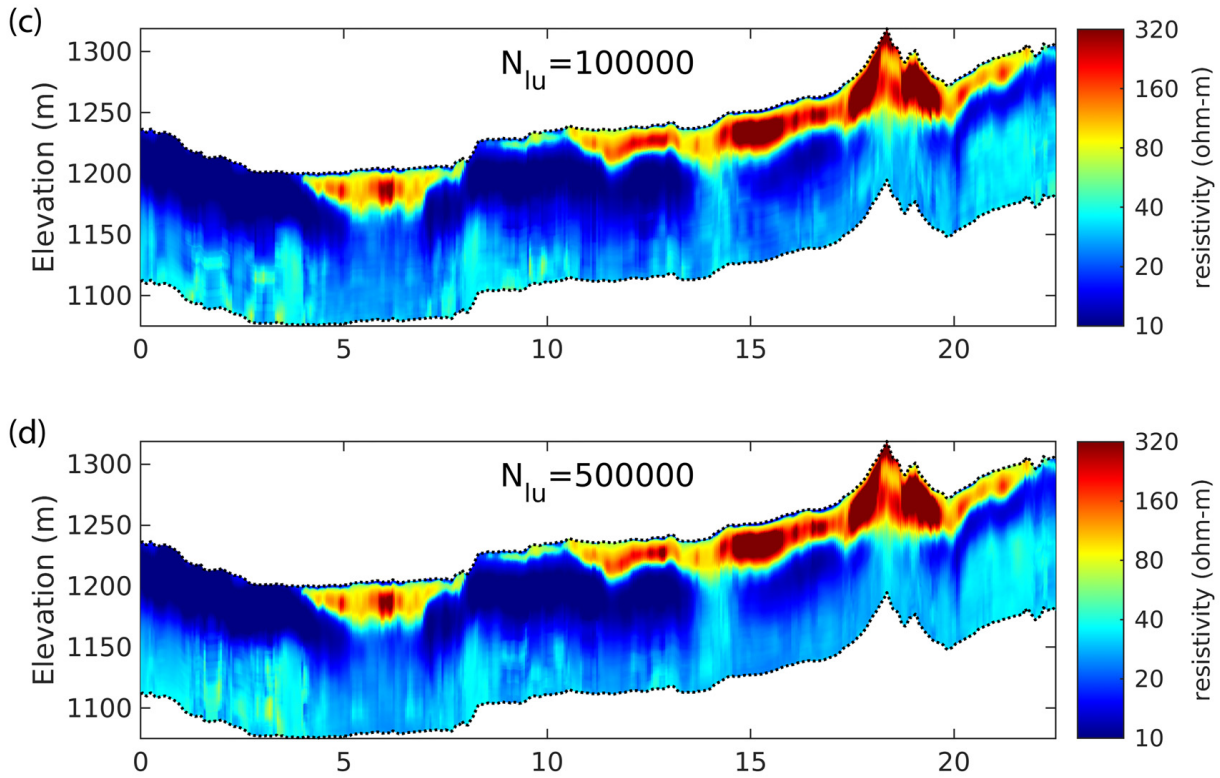

(d)

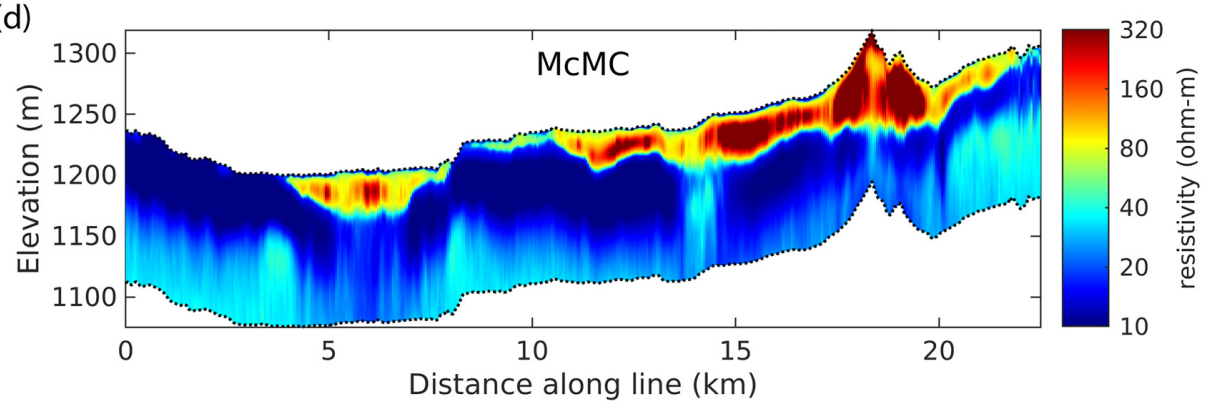

Figure 5. (a)-(d) Posterior mean obtained using $N_{\mathrm{lu}}=[1000,10000,100000,500000]$ accounting for the modelling error, and (e) McMC Metropolis sampling. Compare to Fig. 3 without modelling error.

content, and hence lead to a smoother mean model. But, as investigated here, no biases are apparently introduced. Thus, the results suggest that when making use of the modelling error one can get approximate results, without any bias.
The computation time needed to generate a posterior sample at all 451 locations was $t=[2.7,19,151,724] \mathrm{s}$ in case using $N_{\mathrm{lu}}=[1000$, $10000,100000,500000]$, compared to the $518619 \mathrm{~s}$ (144 hr) needed to run the extended Metropolis algorithm. The results illustrate that 
(a)

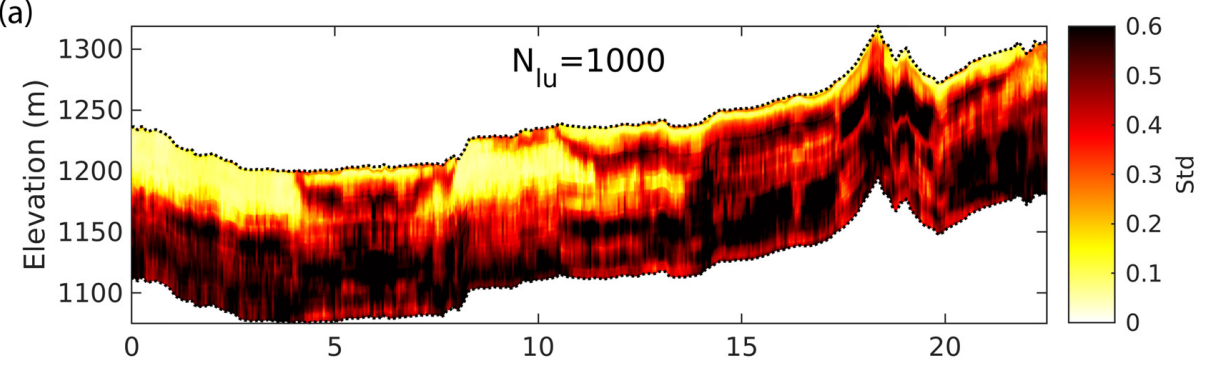

(b)

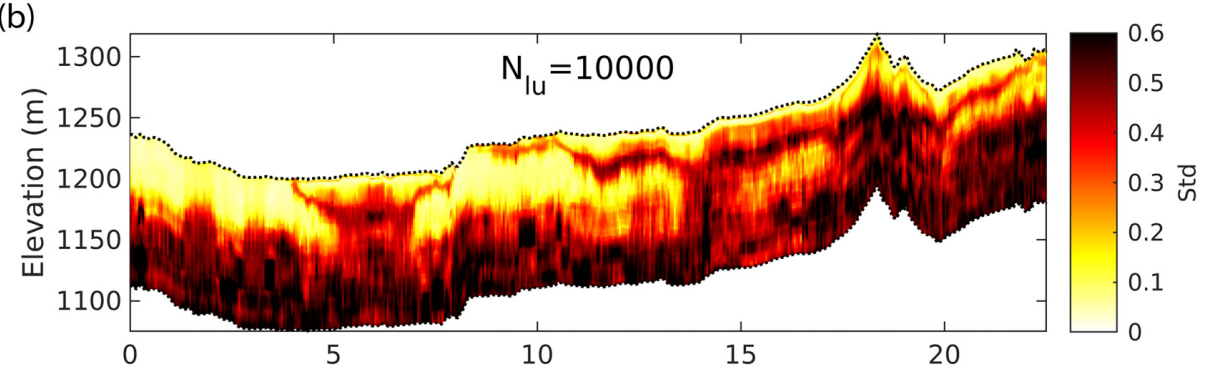

(c)

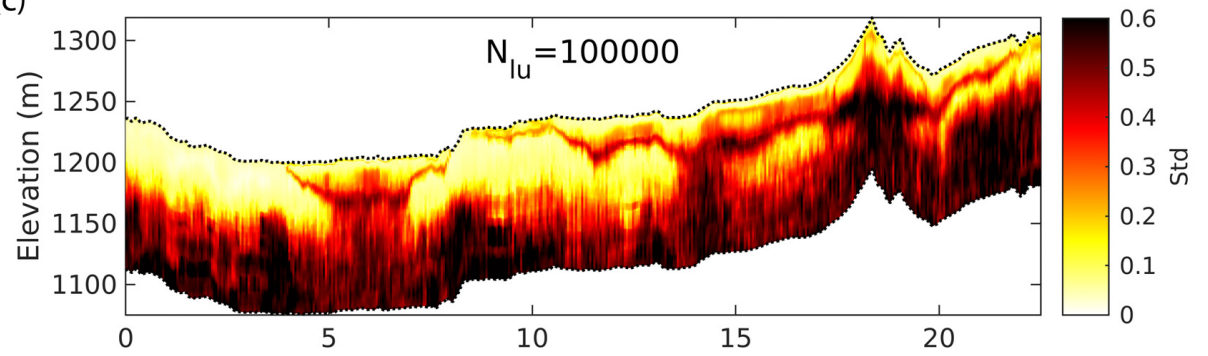

(d)

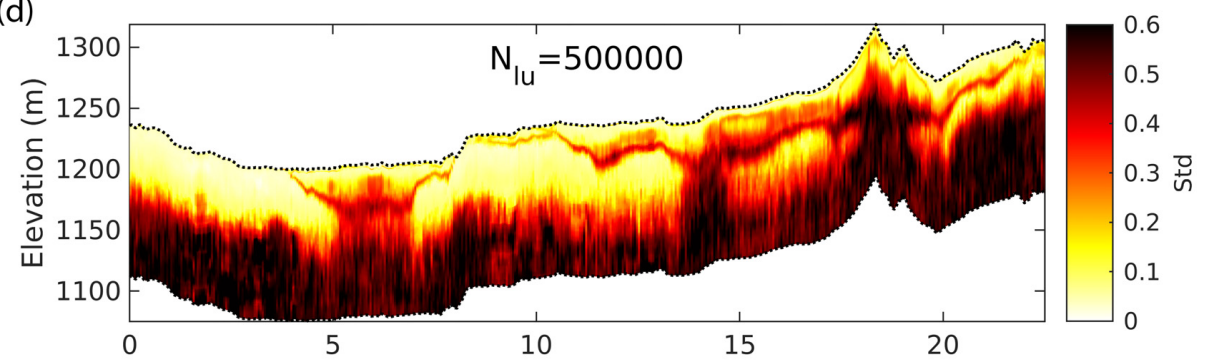

(d)

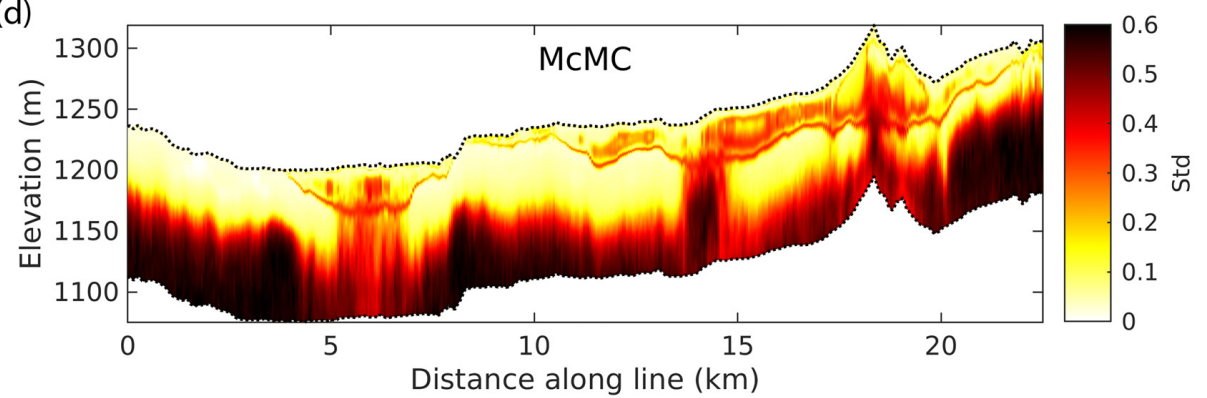

Figure 6. (a)-(d) Posterior standard deviation obtained using $N_{\mathrm{lu}}=[1000,10000,100000,500000]$ accounting for the modelling error, and (e) McMC Metropolis sampling. Compare to Fig. 4 without modelling error.

accurate statistics of the posterior distribution, with a small drop in resolution, can be obtained using the extended rejection sampler with lookup tables, in a very small fraction of the time needed for running the extended Metropolis algorithm. It also illustrates that close to the full posterior distribution can be sampled efficiently using extended rejection sampler using larger training data sets orders of magnitude faster than using the Metropolis sampling algorithm. 
(a) Using modeling error

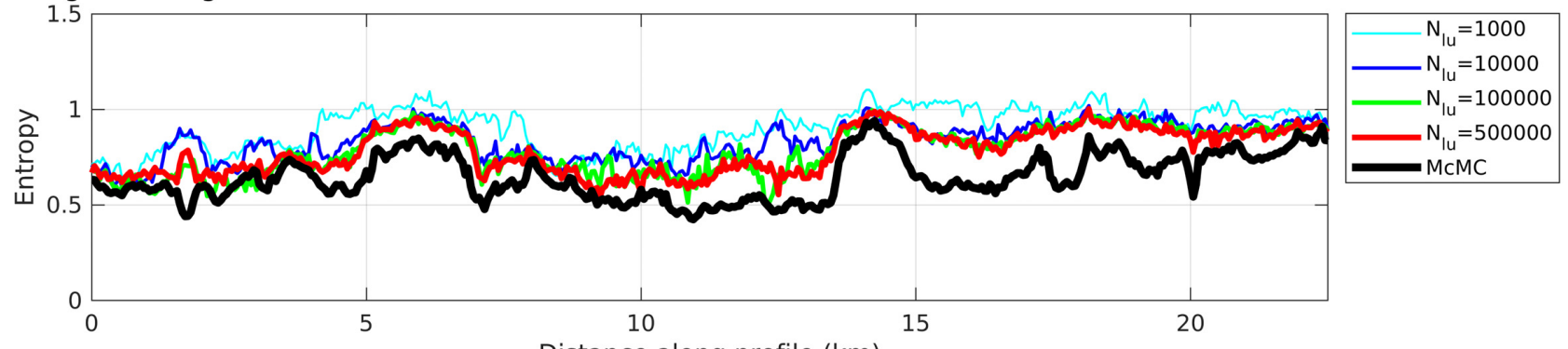

(b) Ignoring modeling error

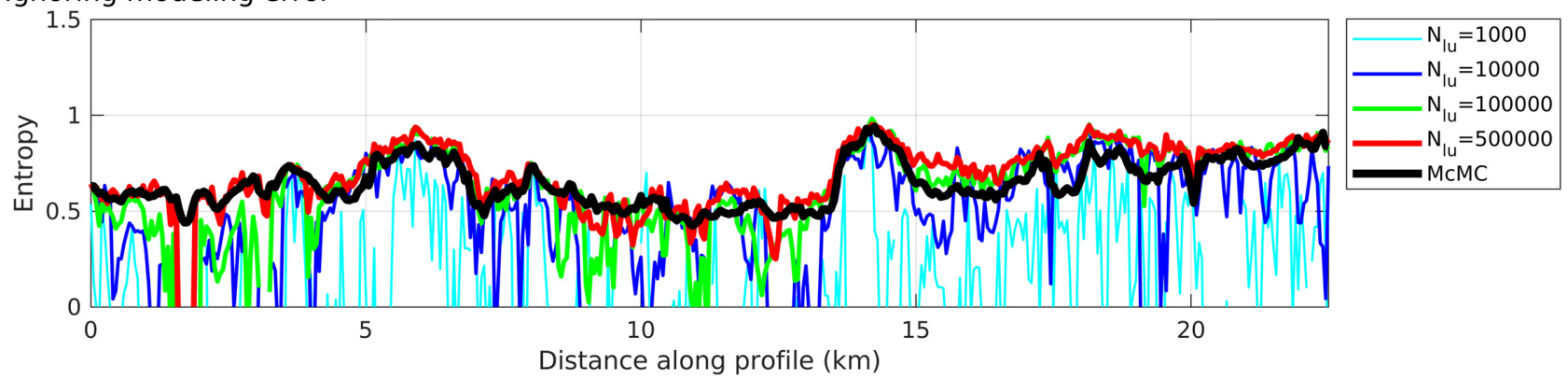

Figure 7. The mean entropy at each $x$-location along the profile in case (a) accounting for modelling errors, and (b) ignoring modelling errors.

(a)

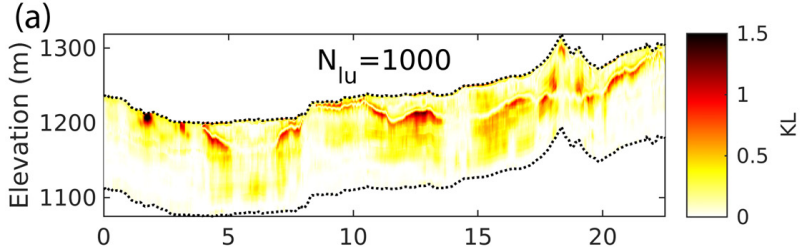

(b)

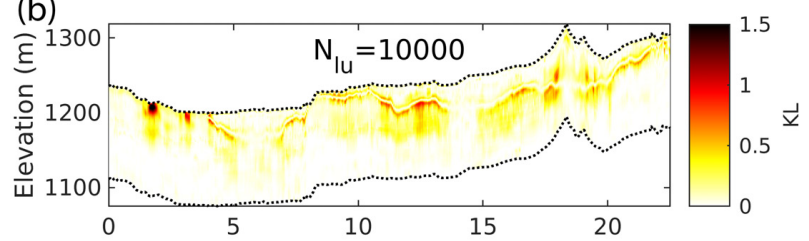

(c)

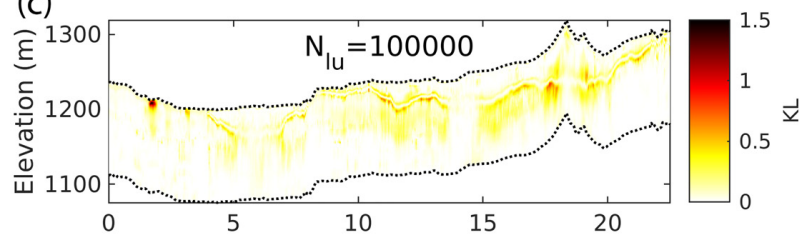

(d)

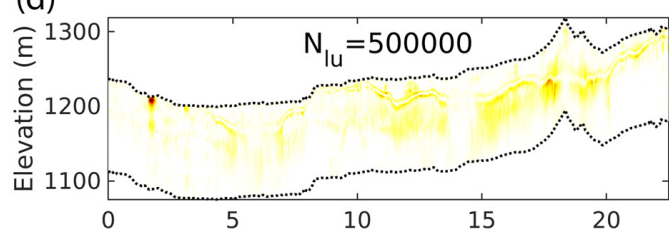

(e)

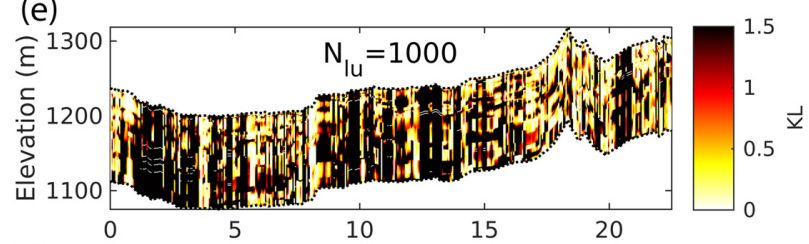

(f)

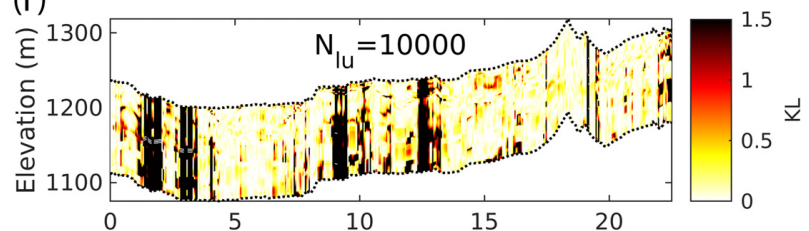

(g)

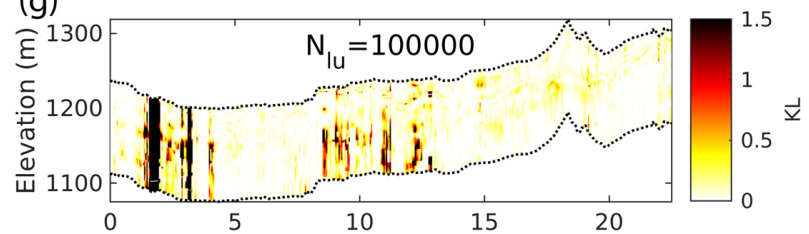

(h)

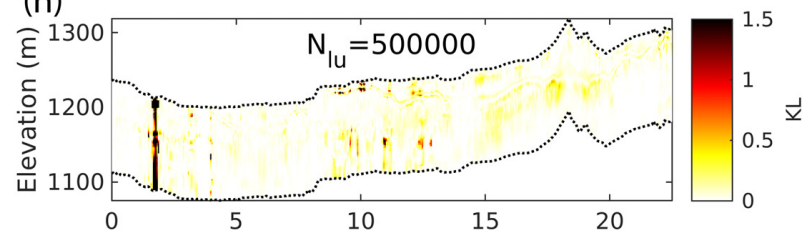

Figure 8. KL distance between the pointwise Gaussian posterior distribution obtained using the extended Metropolis sampler, $\sigma_{\mathrm{mcmc}}(\mathbf{m})$, and the using the extended rejection sampler with lookup tables, $\sigma_{l u}(\mathbf{m})$, considering $N_{\mathrm{lu}}=[1000,10000,100000,500000]$, in cases (a)-(d) accounting for the modelling error, and $(\mathrm{e})-(\mathrm{h})$ ignoring the modelling error.

\section{DISCUSSION}

The extended rejection sampler with lookup tables is a general approach for sampling the posterior distribution of probabilistically formulated inverse problems. The main requirement for using the method is that one must be able to set up a look table consisting of (1) $N_{\text {lu }}$ realizations of the prior, and (2) $N_{\text {lu }}$ corresponding 'full' forward responses. Once the lookup table exists, one only needs to evaluate the likelihood $N_{\text {lu }}$ times to solve the inverse problem.

While the method can be used on any inverse problem, it will make the most sense in case (1) the prior has high information 


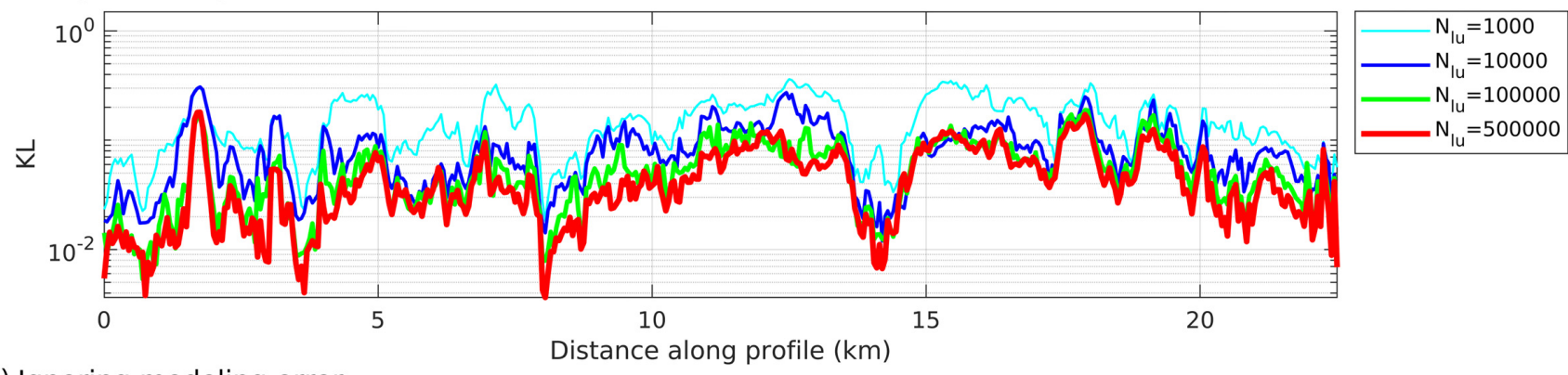

(b) Ignoring modeling error

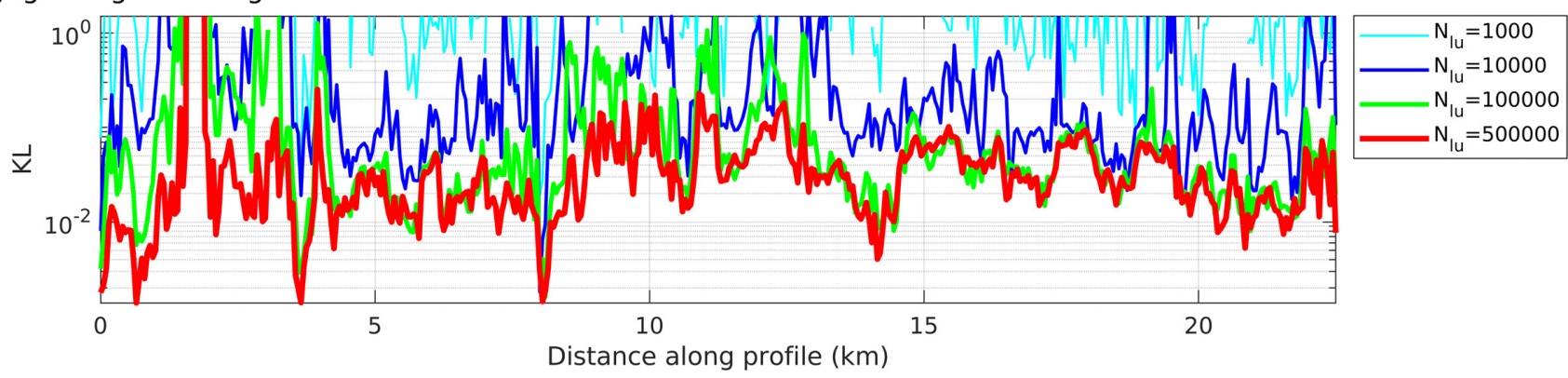

Figure 9. The mean KL distance (between the pointwise Gaussian posterior distribution obtained using the extended Metropolis sampler) at each $x$-location in Fig. 8.

content (otherwise, an approximation to the posterior distribution will be sampled), and/or (2) the inverse problem can be localized such the training data set can be reused many times.

The accuracy and resolution of the method depend on the size of the lookup table relative to the information content of the prior. A prior with less information content (such as a uniform distributes prior) will require a larger lookup table than using a prior with higher information content. Either way, a finite-size lookup table will lead to a limit to the resolution than one can expect. This resolution limit can be quantified and mapped into a modelling error that can be taken into account. Accounting for the modelling error with smaller sized lookup tables, leads to sampling from an approximation to the full posterior distribution, with less information content (higher entropy), but with no apparent inconsistencies. Whether the loss in information is problematic depends on the specific application and use of the inversion results. For the case from Morill, using $N_{\mathrm{lu}}=$ 10000 (Figs 5b and 6b), produce results close to the those obtained using McMC (Figs 5e and 6e), at a tiny fraction of the needed CPU time.

\subsection{Deterministic versuss probabilistic}

Deterministic inverse methods are still more widely applied than probabilistic inverse method. This applies also to the inversion of EM data (Siemon et al. 2009; Auken et al. 2014). One obvious explanation for this is that sampling methods have traditionally been computationally demanding. The sampling methods described by Minsley (2011), Foks \& Minsley (2020) and Hansen \& Minsley (2019) require up to $10 \mathrm{~min}$ to sample the posterior distribution of one EM sounding, while deterministic methods will take less than a second. However, with the examples above the computation time using the extended rejection sampler with lookup tables, can be as low as a few seconds, and considerably lower if one is satisfied with an approximate sample of the posterior distribution, and hence closing the gap in computational demands between deterministic and probabilistic inversion of EM data. Using a smaller lookup table the posterior uncertainty will be slightly higher. Still, a key benefit of the probabilistic approach is that it allows using arbitrarily complex prior information and that the full space of uncertainty is quantified, which should allow for a better description of uncertainty, and hence a better basis for propagating the uncertainty further into for example groundwater models.

\subsection{Computational improvements}

Once a training data set has been computed and stored in a lookup table, the example above suggests that even very large airborne EM surveys with many thousands of soundings, could be probabilistically inverted on a regular laptop of 2020.

Further, the extended rejection sampler with lookup tables is an example of an 'embarrassingly simple parallelization' problem, both related to setting up the lookup table, computation of the likelihood, and computation of posterior statistics. Parallelization is as simple as splitting groups of independent tasks to different threads.

Another approach is to parallelize the sampling of the posterior for each sounding. None of such parallelization has been considered to obtain the results presented here. Therefore, a close to linear relation between CPU speedup and the number of CPU cores available is expected. This is particularly useful as multicore CPUs are becoming quite frequent.

\subsection{Metropolis sampling versus rejection sampling}

In the examples considered above the results obtained using the extended rejection sampler with lookup tables were compared to the results obtained using the extended Metropolis algorithm, which was uses as a reference solution. However, one could argue that the reference should have been that of using the extended rejection sampler with the largest lookup table, $N_{l}=500000$. 
Variants of the Metropolis algorithm samples the posterior through a random walk. This may be very efficient to sample models in the vicinity of each other with high posterior probability. However, it can be very difficult to move between models that are located far from each other separated by models with low posterior probability. It may also be computational demanding using the Metropolis algorithm to sample a subspace of equally possible models, located along some non-trivial-shaped subspace of model parameter space. As discussed initially gradient-based sampling methods have been proposed to alleviate this (Neal et al. 2011; Martin et al. 2012), but these methods cannot readily be used with the extended Metropolis sampler.

Also, the Metropolis algorithm provides a set of realizations that are correlated, due to the use of the random walk. Analysing how many of these realizations are independent is non-trivial (Kass et al. 1998; Ripley 2009; Vats et al. 2015).

The rejection sampler has none of these issues, and will freely move all over the model parameter space, at least as much of the space that is spanned by the proposed models (or the models in the lookup table). Further, the rejection sampler generates independent realizations by construction, removing the need for non-trivial dependency analysis.

For relative low-dimensional problems such as the one considered above, it can, therefore, be argued that the use of the extended rejection sampler with a relatively large lookup table, is preferable to using variants of the Metropolis algorithms, both in terms of the quality of the realizations being generated and certainly in computational demands.

\subsection{Applications for localized inverse problems}

In the example above, only a 1-D prior model (consisting of 125 model parameters) and a 1-D forward model is considered. But, even if the prior is 2-D/3-D one can select a subset of the full model (determined by the correlation of the prior model), and infer information about subsurface resistivity distribution at the location of each data set, independent of other data. In this case, summary statistics of the posterior should be similar to those obtained from full 2-D/3-D inversion.

Another example of a localized inverse problem is that of inversion of reflection seismic amplitude versus offset data as discussed and utilized by Jullum \& Kolbjørnsen (2016) and Hansen \& Jakobsen (2018). In seismic inversion using the convolution method, data at one location in depth is sensitive only to the elastic parameters in the vicinity of the data location, as defined by the prior model, noise model, and the wavelet used for convolution. This means that information about each model parameter can be determined independently of other model parameters by inverting using a small neighbourhood around the model parameter. Jullum \& Kolbjørnsen (2016) demonstrate another approach that takes advantage of the localized property to compute pointwise statistics of the posterior distribution, using a numerically very efficient algorithm.

\subsection{Flexible choice of prior}

The extended rejection sampler can make use of any prior model that can be sampled. For example, in the example given above the model parameters represent log-resistivity. One could use a mapping function that translates resistivity into lithology (as demonstrated by Barfod et al. 2016) which would allow inversion directly for lithological model parameters, with no change to the core of the extended rejection sampling with lookup tables algorithm.

\section{CONCLUSION}

A novel method for localized probabilistic inversion method, using extended rejection sampling with lookup tables, has been proposed. For specific inverse problems, realizations and statistics from the posterior distribution can be computed several orders of magnitudes faster, compared to using McMC methods such as the extended Metropolis algorithm.

An example of 1-D inversion of airborne EM data suggests that large surveys of EM data can be efficiently performed on a laptop using the extended rejection sampler with lookup tables, while the use of McMC sampling methods would require access to a supercomputer.

\section{ACKNOWLEDGEMENTS}

This work is related to a grant (no. 7017-00160B) from the Independent Research Fund Denmark. The airborne EM data used in this study is freely available (Smith et al. 2010) and can be accessed through https://doi.org/10.3133/ofr20101259. The author thanks Thomas Bodin and Klaus Mosegaard whose reviews significantly improved the manuscript.

\section{REFERENCES}

Abraham, J.D., Cannia, J.C., Bedrosian, P.A., Johnson, M.R., Ball, L.B. \& Sibray, S.S., 2012. Airborne Electromagnetic Mapping of the Base of Aquifer in Areas of western Nebraska, U.S. Geological Survey Scientific Investigations Report 2011-5219, p. 38.

Auken, E. et al., 2014. An overview of a highly versatile forward and stable inverse algorithm for airborne, ground-based and borehole electromagnetic and electric data, Explor. Geophys., 46(3), 223-235.

Barfod, A., Møller, I. \& Christiansen, A., 2016. Compiling a national resistivity atlas of Denmark based on airborne and ground-based transient electromagnetic data, J. appl. Geophys, 134, 199-209.

Bosch, M., Mukerji, T. \& Gonzalez, E.F., 2010. Seismic inversion for reservoir properties combining statistical rock physics and geostatistics: a review, Geophysics, 75(5), 75A165-75A176.

Constable, S.C., Parker, R.L. \& Constable, C.G., 1987. Occam's inversion: a practical algorithm for generating smooth models from electromagnetic sounding data, Geophysics, 52(3), 289-300.

Conway, D., Alexander, B., King, M., Heinson, G. \& Kee, Y., 2019. Inverting magnetotelluric responses in a three-dimensional earth using fast forward approximations based on artificial neural networks, Comput. Geosci., 127, $44-52$.

Cordua, K.S., Hansen, T.M. \& Mosegaard, K., 2012. Monte Carlo fullwaveform inversion of crosshole gpr data using multiple-point geostatistical a priori information, Geophysics, 77(2), H19-H31.

De Pasquale, G., Linde, N., Doetsch, J. \& Holbrook, W., 2019. Probabilistic inference of subsurface heterogeneity and interface geometry using geophysical data, Geophys. J. Int., 217(2), 816-831.

Deutsch, C.V. \& Journel, A.G., 1992. GSLIB: Geostatistical Software Library and User's Guide, Oxford Univ. Press.

Earl, D.J. \& Deem, M.W., 2005. Parallel tempering: Theory, applications, and new perspectives, Physical Chemistry Chem. Phys., 7(23), 3910-3916.

Fichtner, A., Zunino, A. \& Gebraad, L., 2018. Hamiltonian Monte Carlo solution of tomographic inverse problems, Geophys. J. Int., 216(2), 13441363. 
Foks, N. \& Minsley, B., 2020. Geophysical Bayesian inference in Python (GeoBiPy). Available at: https://github.com/usgs/geobipy, (accessed August 1, 2020), doi: 10.5066/P9K3YH9O.

Hansen, H.J. \& Jakobsen, A.F., 2018. Local probabilistic inversion of seismic avo data, in 80th EAGE Conference \& Exhibition 2018 Workshop Programme, cp-556-00015, European Association of Geoscientists \& Engineers, https://doi.org/10.3997/2214-4609.201801888.

Hansen, P.C. \& O'Leary, D.P., 1993. The use of the 1-curve in the regularization of discrete ill-posed problems, SIAM jJ. Sci. Comput., 14(6), $1487-1503$.

Hansen, T.M., 2020. Entropy and information content of geostatistical models, Math. Geosci., 1-22.

Hansen, T.M. \& Cordua, K.S., 2017. Efficient monte carlo sampling of inverse problems using a neural network-based forwardapplied to gpr crosshole traveltime inversion, Geophys. J. Int., 211(3), $1524-1533$.

Hansen, T.M. \& Minsley, B.J., 2019. Inversion of airborne EM data with an explicit choice of prior model, Geophys. J. Int., 218(2), 1348-1366.

Hansen, T.M., Cordua, K.C. \& Mosegaard, K., 2012. Inverse problems with non-trivial priors - efficient solution through sequential Gibbs sampling, Comput. Geosci., 16(3), 593-611.

Hansen, T.M., Cordua, K.S., Jacobsen, B.H. \& Mosegaard, K., 2014. Accounting for imperfect forward modeling in geophysical inverse problems-exemplified for crosshole tomography, Geophysics, 79(3), H1$\mathrm{H} 21$.

Hansen, T.M., Cordua, K.S., Zunino, A. \& Mosegaard, K., 2016. Probabilitsic integration of geoinformation, in Joint Inversion, ed. Moorekamp, M., Am. Geophys. Un., Pages 93-116,

Hastings, W., 1970. Monte Carlo sampling methods using Markov chains and their applications, Biometrika, 57(1), 97. doi : 10.2307/2334940.

Hoffman, M.D. \& Gelman, A., 2014. The no-u-turn sampler: adaptively setting path lengths in hamiltonian monte carlo., J. Mach. Learn. Res., 15(1), 1593-1623.

Jullum, M. \& Kolbjørnsen, O., 2016. A gaussian-based framework for local bayesian inversion of geophysical data to rock properties, Geophysics, 81(3), R75-R87.

Kass, R.E., Carlin, B.P., Gelman, A. \& Neal, R.M., 1998. Markov chain Monte Carlo in practice: a roundtable discussion, Am. Stat., 52(2), 93100 .

Köpke, C., Irving, J. \& Elsheikh, A.H., 2018. Accounting for model error in Bayesian solutions to hydrogeophysical inverse problems using a local basis approach, Adv. Water Res., 116, 195-207.

Mariethoz, P. \& Caers, P., 2014. Multiple-point Geostatistics: Stochastic Modeling with Training Images, Wiley.

Martin, J., Wilcox, L.C., Burstedde, C. \& Ghattas, O., 2012. A stochastic Newton MCMC method for large-scale statistical inverse problems with application to seismic inversion, SIAM J. Sci. Comput., 34(3), A1460A1487.

Menke, W., 2012. Geophysical Data Analysis: Discrete Inverse Theory, Vol. 45, Academic Press.
Metropolis, N., Rosenbluth, M., Rosenbluth, A., Teller, A. \& Teller, E., 1953. Equation of state calculations by fast computing machines, J. Chem. Phys., 21, 1087-1092.

Minsley, B.J., 2011. A trans-dimensional Bayesian Markov chain Monte Carlo algorithm for model assessment using frequency-domain electromagnetic data, Geophys. J. Int., 187(1), 252-272.

Moghadas, D., Behroozmand, A.A. \& Christiansen, A.V., 2020, . Soil electrical conductivity imaging using a neural network-based forward solver: applied to large-scale Bayesian electromagnetic inversion, J. appl. Geophys., 176, p. 104012.

Mosegaard, K. \& Tarantola, A., 1995. Monte Carlo sampling of solutions to inverse problems, J. geophys. Res, 100(B7), 12431-12447.

Mosegaard, K., \& Tarantola, A., 2002. Probabilistic approach to inverse problems, in International Handbook of Earthquake and Engineering Seismology, Vol. 81A, chap. 16, pp. 237-265, eds Lee, W., Kanamori, H., Jennings, P., Kisslinger, C., Lee, W.H.K. et al., Academic Press.

Neal, R.M. et al. MCMC using Hamiltonian dynamics. in Handbook of Markov Chain Monte Carlo, Vol. 2(11), p. 2, Chapter 5, Edited by Steve Brooks, Andrew Gelman, Galin Jones, and Xiao-Li Meng. Chapman \& Hall / CRC Press, 2011.

Plessix, R.-E., 2006. A review of the adjoint-state method for computing the gradient of a functional with geophysical applications, Geophys. J. Int., 167(2), 495-503.

Ripley, B.D., 2009. Stochastic Simulation, Vol. 316, John Wiley \& Sons.

Sambridge, M., 2013. A parallel tempering algorithm for probabilistic sampling and multimodal optimization, Geophys. J. Int., 196(1), 357-374.

Siemon, B., Christiansen, A.V. \& Auken, E., 2009. A review of helicopterborne electromagnetic methods for groundwater exploration, Near Surf. Geophys., 7(5-6), 629-646.

Smith, B.D., Abraham, J.D., Cannia, J.C., Minsley, B.J., Deszcz-Pan, M. \& Ball, L.B., 2010. Helicopter electromagnetic and magnetic geophysical survey data, portions of the North Platte and South Platte Natural Resources Districts, Western Nebraska, Tech. Rep. 2010-1259, U.S. Geological Survey Scientific Investigations Report.

Tarantola, A., 1986. A strategy for nonlinear elastic inversion of seismic reflection data, Geophysics, 51(10), 1893-1903.

Tarantola, A., 2005. Inverse Problem Theory and Methods for Model Parameter Estimation, SIAM.

Tarantola, A. \& Valette, B., 1982a. Generalized nonlinear inverse problems solved using the least squares criterion, Rev. Geophys. Space Phys., 20(2), 219-232.

Tarantola, A. \& Valette, B., 1982b. Inverse problems= quest for information, J. geophys, 50(3), 150-170.

Tromp, J., Tape, C. \& Liu, Q., 2005. Seismic tomography, adjoint methods, time reversal and banana-doughnut kernels, Geophys. J. Int., 160(1), 195216

Vats, D., Flegal, J.M. \& Jones, G.L., 2015. Multivariate output analysis for Markov Chain Monte Carlo, preprint (arXiv:1512.07713).

Zunino, A., Mosegaard, K., Lange, K., Melnikova, Y. \& Hansen, T.M., 2014. Monte Carlo reservoir analysis combining seismic reflection data and informed priors, Geophysics, 80(1), R31-R41. 